\title{
Effective drifts in dynamical systems with multiplicative noise: a review of recent progress
}

\author{
Giovanni Volpe ${ }^{1}$ and Jan Wehr ${ }^{2}$ \\ 1 Soft Matter Lab, Department of Physics, and UNAM-National Nanotechnology Research Center, \\ Bilkent University, Ankara 06800, Turkey \\ 2 Department of Mathematics and Program in Applied Mathematics, University of Arizona, Tucson, \\ Arizona 85721, USA \\ E-mail: giovanni.volpe@fen.bilkent.edu.trandwehr@math.arizona.edu \\ Invited by Maciej Lewenstein
}

Received 4 July 2014, revised 8 December 2015

Accepted for publication 6 January 2016

Published 18 April 2016

\begin{abstract}
Noisy dynamical models are employed to describe a wide range of phenomena. Since exact modeling of these phenomena requires access to their microscopic dynamics, whose time scales are typically much shorter than the observable time scales, there is often need to resort to effective mathematical models such as stochastic differential equations (SDEs). In particular, here we consider effective SDEs describing the behavior of systems in the limits when natural time scales become very small. In the presence of multiplicative noise (i.e. noise whose intensity depends upon the system's state), an additional drift term, called noise-induced drift or effective drift, appears. The nature of this noise-induced drift has been recently the subject of a growing number of theoretical and experimental studies. Here, we provide an extensive review of the state of the art in this field. After an introduction, we discuss a minimal model of how multiplicative noise affects the evolution of a system. Next, we consider several case studies with a focus on recent experiments: the Brownian motion of a microscopic particle in thermal equilibrium with a heat bath in the presence of a diffusion gradient; the limiting behavior of a system driven by a colored noise modulated by a multiplicative feedback; and the behavior of an autonomous agent subject to sensorial delay in a noisy environment. This allows us to present the experimental results, as well as mathematical methods and numerical techniques, that can be employed to study a wide range of systems. At the end we give an application-oriented overview of future projects involving noise-induced drifts, including both theory and experiment.
\end{abstract}

Keywords: dynamical systems, multiplicative noise, time-scale competition, delayed feedback, effective drifts

(Some figures may appear in colour only in the online journal) 


\section{Introduction}

Dynamical systems are widely employed to describe and predict the behavior of complex phenomena [1]. At any given time $t$, a dynamical system is characterized by a state $x_{t}$, which evolves according to a deterministic rule. A complete deterministic description requires access to the system's microscopic dynamics. A classical example is the Brownian motion of a particle in a fluid [2]. The motion of the particle and fluid molecules is deterministic, as it can be described by a set of Newton's equations: knowing the initial positions and velocities of all particles (i.e. the Brownian particle and the fluid molecules), it is in principle possible to determine their motion over time, as is done in the molecular dynamics simulation shown in figure 1(a). Nevertheless, the resulting motion of the microscopic particle (shown in figure 1(b)) appears to be random, especially if one has no access to the exact positions and velocities of the fluid molecules. In fact, it is often an impossible task to construct a model for a dynamical system that accounts for its microscopic dynamics. For example, even though in principle it would be possible to construct a model of Brownian motion writing down Newton's equation of motion for the particle as well as for each fluid molecule, this is a practically unfeasible task due to the huge number of molecules in any realistic situation-of the order of the Avogadro number $6.02 \cdot 10^{23}$.

It is often convenient to reduce the effective number of degrees of freedom in order to obtain more tractable models. This can be achieved by introducing some randomness. For example, the Brownian motion of a particle can be modelled by the stochastic differential equation (SDE)

$$
\mathrm{d} x_{t}=\sigma \mathrm{d} W_{t},
$$

where $W_{t}$ is a Wiener process, i.e. a stochastic process with continuous paths, whose increments $W_{t}-W_{s}$ are independent and normally distributed with mean zero and variance $t-s$ [3], representing the stochastic driving, $\sigma=\sqrt{2 D_{\mathrm{SE}}}$, and $D_{\mathrm{SE}}$ is the Stokes-Einstein diffusion constant [2]. The resulting Brownian motion is shown in figure 2(a) for the case of a $1 \mu \mathrm{m}$-radius Brownian particle in water at room temperature in bulk, i.e. far away from any boundaries. SDE (1) is arguably the simplest way to describe the properties of a free diffusion, as it only involves explicitly one degree of freedom. The term $\sigma \mathrm{d} W_{t}$ is thus a mathematical model for the noise, which permits one to implicitly account for the microscopic dynamics of the system (in this case, the motion of the fluid molecules). We stress that SDE (1) with an initial condition $x_{0}$ has a unique solution, $x_{t}=x_{0}+\sigma W_{t}$, for any given realization of $W_{t}$. Similar models have been employed to describe a wide range of phenomena, from thermal fluctuations in electronic circuits and evolution of stock prices, to heterogeneous response of biological systems, to stochasticity in gene expression [4-8].

Often, the system's state influences the intensity of the driving noise, as it is fed back on the input noise and modulates it. If such feedback loop is multiplicative, i.e. the intensity of the input noise gets multiplied by a function of the system's state, as shown in figure 2(b), one says that the system is driven by
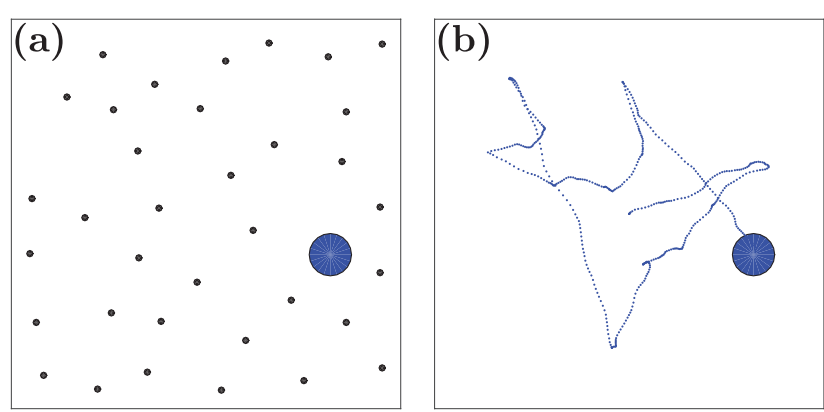

Figure 1. Stochastic motion from deterministic simulations. (a) A microscopic particle (large circle) immersed in a fluid continuously undergoes collisions with the fluid molecules (dots). (b) The resulting motion obtained from a molecular dynamics simulation (dotted line), despite being deterministic, appears to be random, especially if one has no access to the exact positions and velocities of the fluid molecules.

a multiplicative noise. For example, the Brownian fluctuations of a microscopic particle near a wall are reduced by hydrodynamic interactions [9], as shown in figure 2(c). The corresponding SDE (in the absence of other forces, see SDE (4) for a more complete model) is

$$
\mathrm{d} x_{t}=\sigma\left(x_{t}\right) \mathrm{d} W_{t},
$$

where $\sigma\left(x_{t}\right)=\sqrt{2 D_{\perp}\left(x_{t}\right)}$ and $D_{\perp}(x)$ is the particle's diffusion coefficient in the direction normal to the wall, which depends on the particle-wall distance $x$. Let us mention that SDE (2) with the noise coefficient $\sigma$ equal to a power of $|x|$ was studied recently in detail in the context of diffusion in inhomogeneous media; the reader is referred to [10] and [11] for analytical and numerical results, concerning ergodicity, anomalous diffusion and other properties of this model. Similar models are employed to describe, e.g. the change of the step size of a random walk due to inhomogeneity of the medium [12], the alteration of the volatility of a stock price depending on its actual value [13], and the regulation of the stochastic expression of a gene by the concentration of its products [14].

Unlike SDE (1), the integration of SDE (2) has to be performed carefully, because a realization of the Wiener process $W_{t}$ has infinite variation on any interval (in fact, the derivative $\frac{\mathrm{d} W_{t}}{\mathrm{~d} t}$ does not exist anywhere) [3]. The stochastic integral $\int_{0}^{T} f\left(x_{t}\right) \circ_{\alpha} \mathrm{d} W_{t} \equiv \lim _{N \rightarrow \infty} \sum_{n=0}^{N-1} f\left(x_{t_{n}}\right) \Delta W_{t_{n}}$, where $t_{n}=\frac{n+\alpha}{N} T$ and $\alpha$ is a real number (typically, $\alpha=0,0.5$ or 1 ), may have different values for different choices of $\alpha[3,15]$. Therefore, a complete model is defined by an SDE and the integration convention, which must be determined on the basis of the available experimental data or derived from another unambiguous model [16]. If desired, one can change the convention to $\alpha^{\prime}$, but only by adding an appropriate noise-induced drift term at the same time; as we will see in section 2, this noiseinduced drift term is in general proportional to $\sigma\left(x_{t}\right) \frac{\mathrm{d}}{\mathrm{d} x} \sigma\left(x_{t}\right)$ [3]. Thus, a more precise way of writing SDE (2) is

$$
\mathrm{d} x_{t}=\left(\alpha-\alpha^{\prime}\right) \sigma\left(x_{t}\right) \frac{\mathrm{d} \sigma\left(x_{t}\right)}{\mathrm{d} x} \mathrm{~d} t+\sigma\left(x_{t}\right) \circ_{\alpha^{\prime}} \mathrm{d} W_{t},
$$


(a)

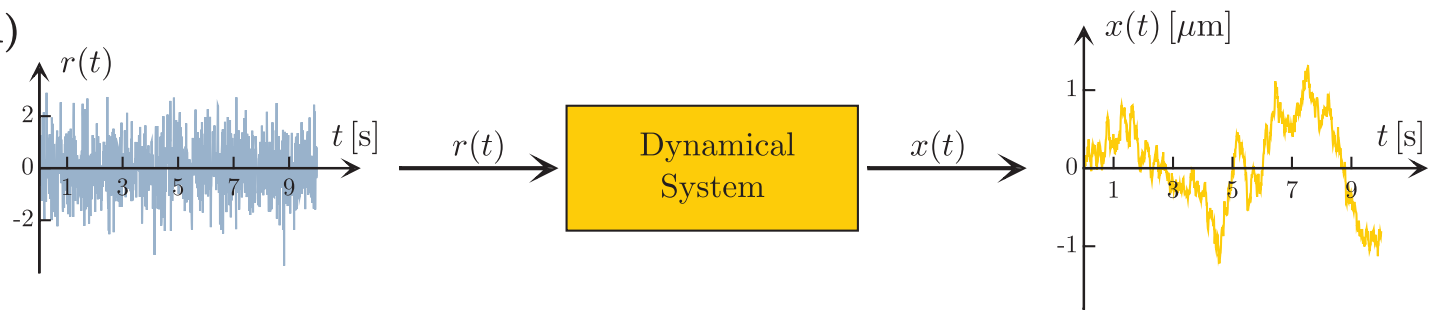

(b)

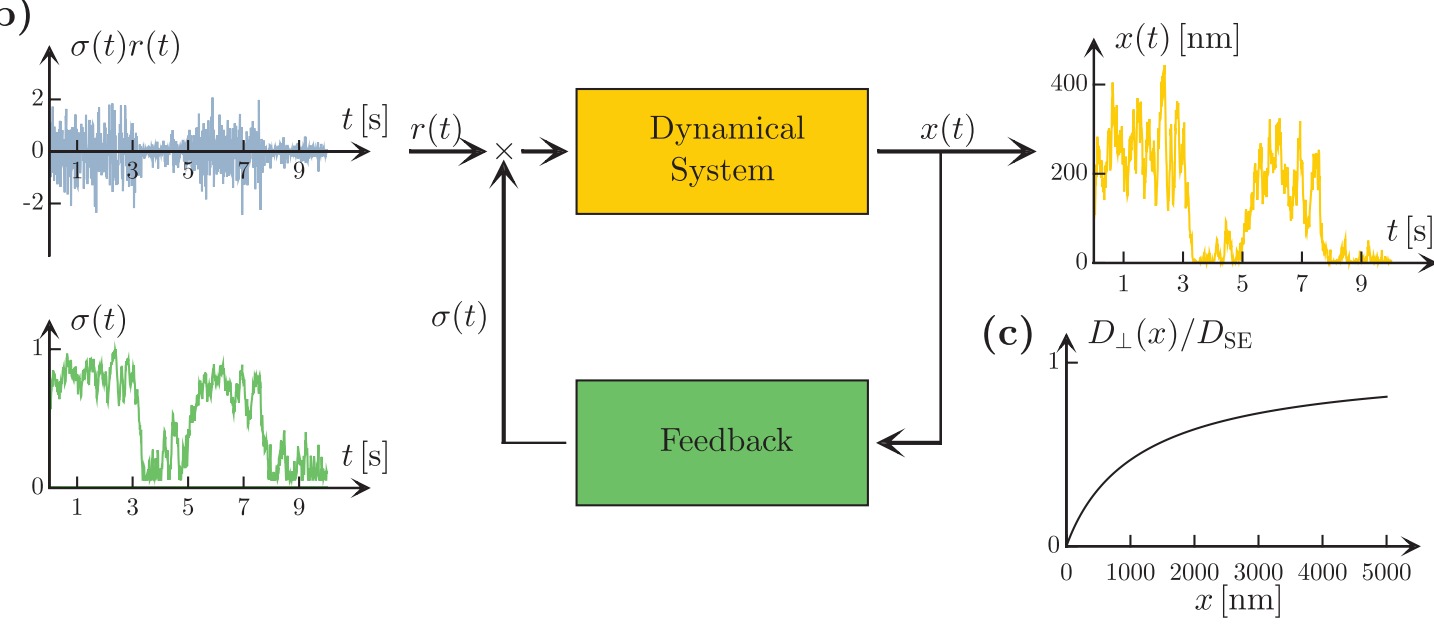

Figure 2. Stochastic dynamical system without and with feedback. (a) A schematic representation of a stochastic dynamical system: the system's state $x(t)$ evolves as the system is driven by a noisy input $r(t)$. (b) Same system with feedback $\sigma(t)=\sigma(x(t))$ : $r(t)$ is now modulated by $\sigma(t)$, and $x(t)$ is clearly affected. The data correspond to the motion of a $1 \mu \mathrm{m}$-radius Brownian particle in water at room temperature (a) in bulk and (b) close to a boundary; the curve in (c) shows the diffusion coefficient $D_{\perp}(x)$ of the particle in the direction perpendicular to the boundary (normalized to the bulk diffusion coefficient $D_{\mathrm{SE}}=D_{\perp}(\infty)$ ) as a function of its distance from the boundary $x$.

where the integration convention indicated by $\alpha^{\prime}$ and the noise-induced drift, i.e. $\left(\alpha-\alpha^{\prime}\right) \sigma\left(x_{t}\right) \frac{\mathrm{d} \sigma\left(x_{t}\right)}{\mathrm{d} y}$, are explicitly shown. This shows that the equations $\mathrm{d} x_{t}=\sigma\left(x_{t}\right) \circ_{\alpha} \mathrm{d} W_{t}$ are not equivalent for different $\alpha$ and it is thus clear that the choice of the appropriate SDE-convention pair is of critical importance from the modeling perspective, especially when the model is employed to predict the system's behavior under new conditions.

Finally, let us note that until now we have only considered equations without a deterministic drift. If a deterministic drift $g\left(x_{t}\right)$ is present, $\operatorname{SDE}(2)$ becomes

$$
\mathrm{d} x_{t}=g\left(x_{t}\right) \mathrm{d} t+\sigma\left(x_{t}\right) \mathrm{d} W_{t} .
$$

However, the presence of $g\left(x_{t}\right) \mathrm{d} t$ does not lead to any ambiguities, since this term can be integrated in a standard way.

In section 2, we introduce the fundamental concepts and ideas in a simple and intuitive way, making use of a minimal discrete-time model. In section 3, we describe in detail some case studies focusing mainly on recent experiments; this allows us to present not only the experimental findings, but also some mathematical methods and numerical techniques that can be employed to study a wide range of systems. Finally, in section 4, we give an overview of various other situations where noise-induced drifts in the limiting SDEs become relevant when describing a system driven by multiplicative noise. We argue that the possibility of such noiseinduced drifts and of their dramatic consequences should be recognized and accounted for in many cases where SDEs with multiplicative noise are routinely employed to predict the behavior and evolution of complex physical, chemical, biological, and economic phenomena.

\section{A minimal discrete-time model}

In this section we introduce a minimal (discrete-time) model to demonstrate how multiplicative noise affects the evolution of a system. We will see, in particular, how the presence of a multiplicative noise can generate a noise-induced drift and alter the long-term probability distribution of the system's state.

We start by considering the system without multiplicative noise described by SDE (1). The continuous-time solution $x(t)$ of SDE (1) can be approximated by a discrete-time sequence $x_{n}$, which is the solution of the corresponding finite-difference equation (FDE) evaluated at regular time steps $t_{n}=n \Delta t$; for $\Delta t$ sufficiently small, $x_{n} \approx x\left(t_{n}\right)$. The finite-difference (FD) terms corresponding to $\mathrm{d} x_{t}$ are $x_{n+1}-x_{n}$, while those corresponding to $\mathrm{d} W_{t}$ are given by a sequence of independent random numbers with zero mean and variance $\Delta t,{ }^{3}$ such as a sequence of indepenendent random numbers with values $\pm \sqrt{\Delta t}$. We thus obtain the discrete-time random walker FDE:

${ }^{3}$ This follows from the properties of a Wiener process $W_{t}$ : $\left\langle\int_{t}^{t+\Delta t} \mathrm{~d} W_{t}\right\rangle=\left\langle W_{\Delta t}\right\rangle=0$ and $\left\langle\left(\int_{t}^{t+\Delta t} \mathrm{~d} W_{t}\right)^{2}\right\rangle=\left\langle W_{\Delta t}^{2}\right\rangle=\Delta t$. A more detailed discussion can be found in [87]. 
(a)

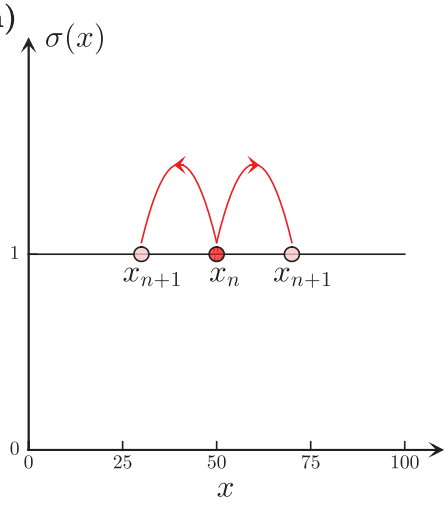

(b)

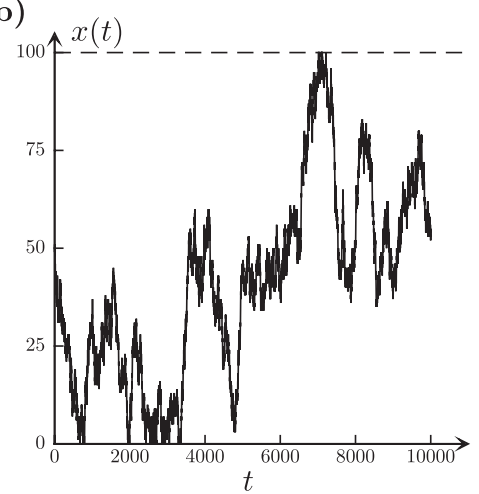

(c)

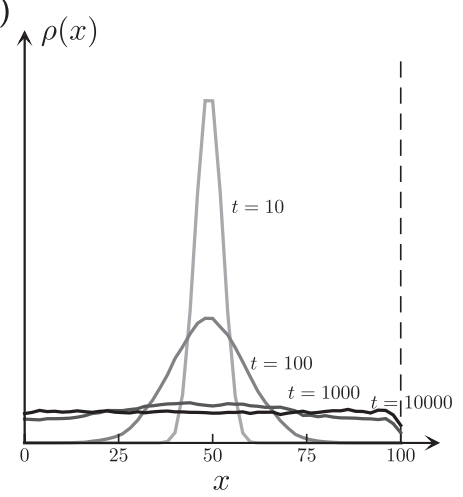

Figure 3. Evolution of the random walker without multiplicative noise described by SDE (5). (a) The intensity of the noise $\sigma$ does not depend on the system's state $x$; therefore at each time step the state increases or decreases by a fixed amount $\sigma \sqrt{\Delta t}$ (not to scale) with equal probability (0.5). (b) Example of a trajectory of the system in state space $(\sigma=1)$. (c) Probability density of the distributions at selected times (calculated from 10000 simulated trajectories). Reflecting boundary conditions are imposed at $x=0$ and $x=100$. Note that the steady-state probability distribution is uniform, as can be expected from the absence of deterministic forces acting on the system.

$$
x_{n+1}=x_{n} \pm \sigma \sqrt{\Delta t},
$$

where the symbol ' \pm ' signifies that at each step the sign is chosen randomly. As shown in figure 3(a), at each time step the value of the system's state either increases or decreases with the same probability $(0.5)$ and amplitude $\sigma \sqrt{(\Delta t)}$. In figure 3(b), we show a simulated trajectory for the evolution of such system starting at $x_{0}=50$. Since the probability and amplitude of the step are equal in both directions (i.e. ' + ' and '-'), the system's state evolves in a symmetric way. In the simulations presented in figure 3 , in order to obtain a steadystate probability distribution for the system's state, we have restricted the system's space to the interval between 0 and 100 , introducing reflecting boundary conditions at $x=0$ and $x=100 .{ }^{4}$ As shown in figure 3(c), we obtain a steady-state probability distribution that is uniform, as can be expected due to the absence of deterministic forces acting on the system [12].

We will now consider the system with multiplicative noise described by SDE (2). Again, we can approximate the continuous-time solution by a discrete-time sequence of states, which solves the corresponding FDE. Now, however, we have to decide: Where should the value of $\sigma(x)$ be evaluated at consecutive time steps? At the starting state $x_{n}$ ? At the final state $x_{n+1}$ ? At the (midpoint) intermediate state $\frac{1}{2}\left(x_{n}+x_{n+1}\right)$ ? At some other state?

Let us first consider the case when the value of $\sigma(x)$ is evaluated at $x_{n}$ (figures 4(a)-(c)), explicitly:

$$
x_{n+1}=x_{n} \pm \sigma\left(x_{n}\right) \sqrt{\Delta t} .
$$

This is particularly convenient from a computational point of view because the value of $x_{n}$ is already available when the FDE is solved iteratively (see also appendix A). As shown in figure 4(a), the value of $x$ either increases or decreases by the same amount, equal to $\sigma\left(x_{n}\right) \sqrt{\Delta t}(\sigma(x)$ is plotted by the black solid line). A numerical solution is shown in figure 4(b) and

${ }^{4}$ In absence of boundary conditions, the state space would be unbounded and a steady state probability distribution would not exist. the evolution of the probability density of the system's state is shown in figure 4(c). At the beginning, the state is $x_{0}=50$ and evolves in a symmetric way, but, as time passes, the system reaches an asymmetric steady-state probability distribution and is more likely found in low-noise states, i.e. states for which $\sigma(x)$ is smaller.

We can also consider the case when the value of $\sigma(x)$ is evaluated at the midpoint state $\frac{1}{2}\left(x_{n}+x_{n+1}\right)$. A heuristic argument for applying this convention to real systems is that the value of $\sigma(x)$ should be averaged over the change of the system's state. In this case, the corresponding FDE is

$$
x_{n+1}=x_{n} \pm \sigma\left(\frac{1}{2}\left(x_{n}+x_{n+1}\right)\right) \sqrt{\Delta t} .
$$

We will explain how to approximately solve this equation below (see equation (10) and appendix A). Figure 4(d) shows that the change of the system's state now becomes asymmetric because it is larger (smaller) when moving toward increasing (decreasing) $\sigma$. A simulated trajectory is shown in figure 4(e) and the evolution of the probability density of the system's state is shown in figure 4(f). At the beginning the probability density drifts towards higher-noise states. However, at long times, the system is still more likely to be found in low-noise states.

Furthermore, for reasons that will become clear later (section 3.1), it can be also useful to evaluate $\sigma$ at other states and, in particular, at the final state $x_{n+1}$. In this case, the corresponding FDE is given by

$$
x_{n+1}=x_{n} \pm \sigma\left(x_{n+1}\right) \sqrt{\Delta t} .
$$

The change in the system's state becomes even more asymmetric than in the previous case (figure $4(\mathrm{~g})$ ) and an even larger noise-induced drift can be seen at short times (figures 4(h) and (i)). Interestingly, the steady-state probability density distribution appears to be uniform, i.e. independent of the value of $\sigma(x)$. We can conclude that in this case the noiseinduced drift is sufficient to compensate for the tendency of the system to linger in low-noise states. 
(a)

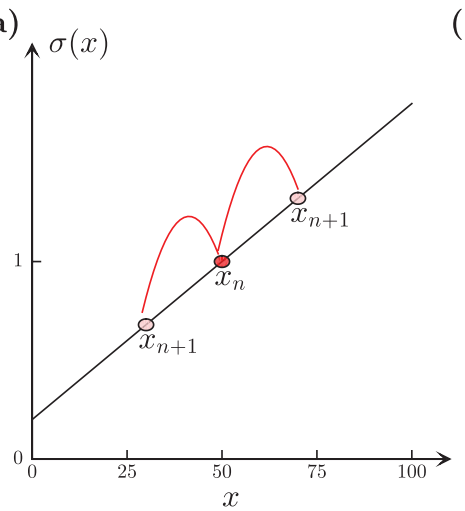

(d)

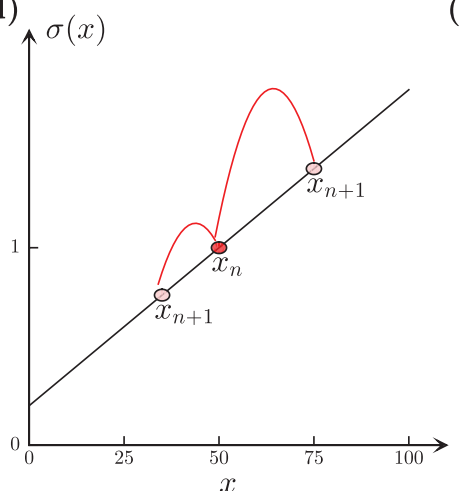

(g)

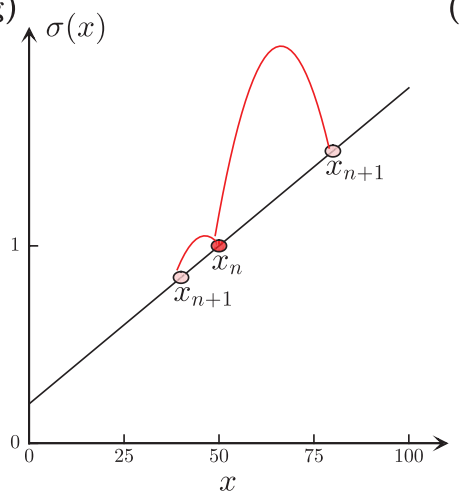

(b)

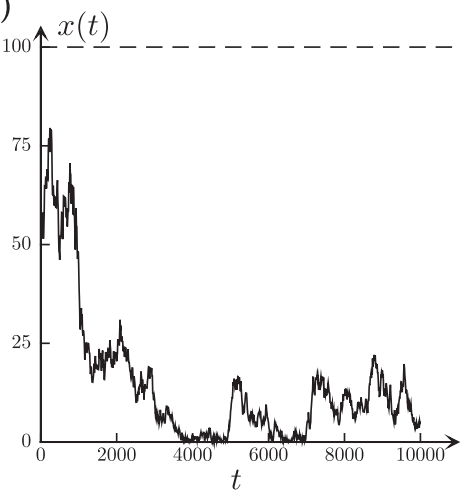

(e)

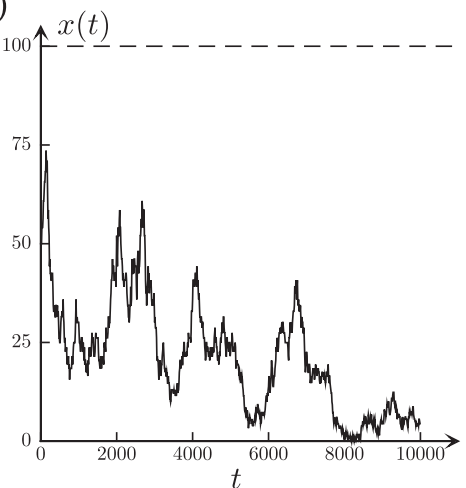

(h)

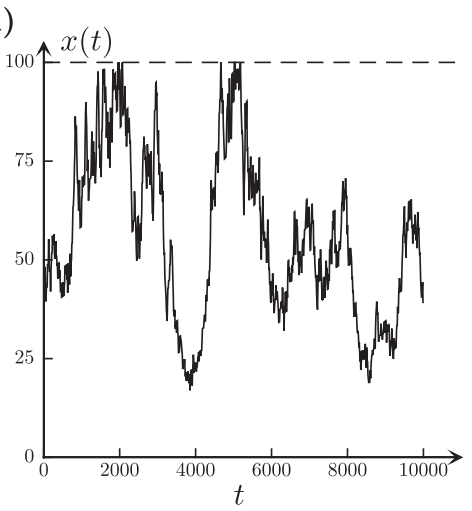

(c)

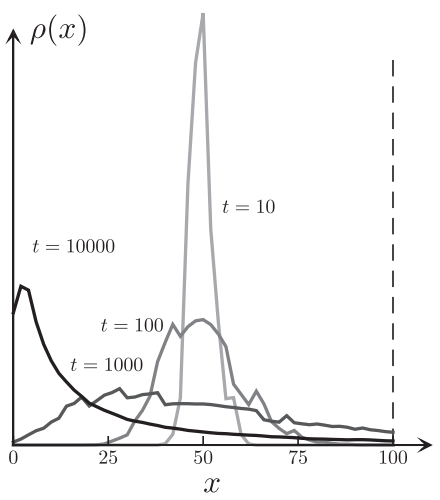

(f)

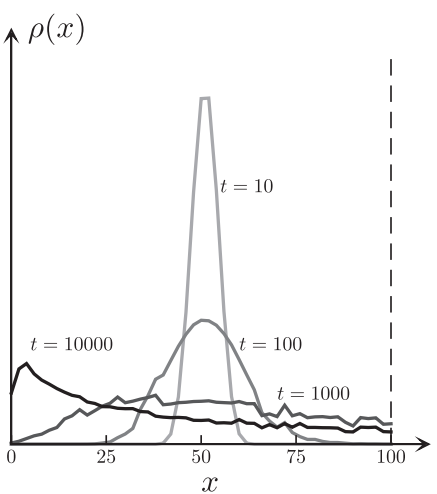

(i)

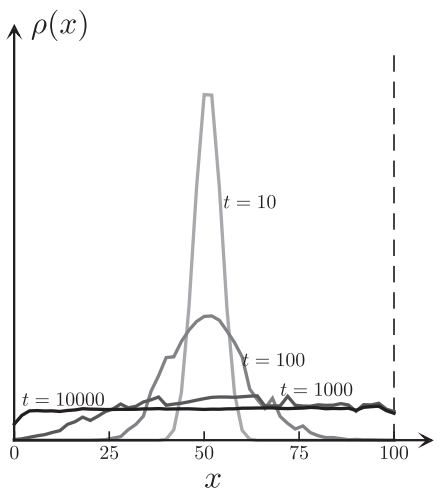

Figure 4. Evolution of the random walker with multiplicative noise described by SDE (9) for various values of $\alpha$. (a) For $\alpha=0$ (equation (6)), the amplitude of each random step is a function of the initial state and is therefore symmetrically distributed; (b) example of a trajectory in state space; (c) probability density of the distributions at selected times. The corresponding results for $\alpha=0.5$ (equation (7)) and $\alpha=1$ (equation (8)) are shown in ((d)-(f)) and ((g)-(i)), respectively. In all cases, reflecting boundary conditions are imposed at $x=0$ and $x=100$. Note that the steady-state probability distribution is uniform only in the $\alpha=1$ case, while in the other two cases it is peaked in the low-noise (small $\sigma(x)$ ) region. The steady-state probability distributions are calculated from 100000 simulated trajectories.

In order to understand the origin of the noise-induced drift and how it is related to the way the noise term is evaluated, we study the following FDE:

$$
x_{n+1}=x_{n} \pm \sigma\left(x_{n}+\alpha \Delta x\right) \sqrt{\Delta t},
$$

where $\Delta x=x_{n+1}-x_{n}$. We expand the factor $\sigma\left(x_{n}+\alpha \Delta x\right)$ as

$$
\sigma\left(x_{n}+\alpha \Delta x\right) \approx \sigma\left(x_{n}\right)+\alpha \frac{\mathrm{d} \sigma\left(x_{n}\right)}{\mathrm{d} x} \Delta x .
$$

Substituting the first-order expansion of $\Delta x \approx \pm \sigma\left(x_{n}\right) \sqrt{\Delta t}$, we obtain

$$
\sigma\left(x_{n}+\alpha \Delta x\right) \approx \sigma\left(x_{n}\right) \pm \alpha \sigma\left(x_{n}\right) \frac{\mathrm{d} \sigma\left(x_{n}\right)}{\mathrm{d} x} \sqrt{\Delta t}
$$

and we can therefore re-write equation (9) as

$$
x_{n+1}=x_{n}+\underbrace{\alpha \sigma\left(x_{n}\right) \frac{\mathrm{d} \sigma\left(x_{n}\right)}{\mathrm{d} x} \Delta t}_{\text {noise-induced drift }} \pm \sigma\left(x_{n}\right) \sqrt{\Delta t} .
$$

Therefore, various values of $\alpha$ lead to different noise-induced drifts and, consequently, to different steady-state probability distributions, as we have seen in figure 4 for the cases $\alpha=0$, 0.5 , and 1 . Importantly, we note that the presence of the noiseiduced drift does not depend on the value of $\Delta t$, i.e. it is present in the limiting SDE, as we will see in the case studies presented in the section 3 .

The parameter $\alpha$ determines how the stochastic integration is performed. Common choices are: the Itô integral with $\alpha=0$ corresponding to the use of the initial value (equation (6)) [17]; 
the Stratonovich integral with $\alpha=0.5$ corresponding to the use of the midpoint value (equation (7)) [18]; and the anti-Itô or isothermal integral with $\alpha=1$ corresponding to the use of the final value (equation (8)) $[19,20]$. In particular, $\alpha=0$ models are typically employed in economics [5] and biology [21] because of their property of 'not looking into the future', referring to the fact that, when the integral is approximated by a sum, the first point of each interval is used (see also appendix A). $\alpha=0.5$ naturally emerges in physical systems with noise correlation time $\tau>0$, e.g. the SDEs describing electrical circuits driven by a multiplicative noise [22]; this is explained mathematically by the Wong-Zakai theorem, which states that, if in SDE (2) the Wiener process is approximated by a sequence of smooth processes with symmetric covariance and $\tau$ going to 0 , the resulting limiting SDE should be interpreted according to Stratonovich calculus [23]. Finally, $\alpha=1$ naturally emerges in physical systems in equilibrium with a heat bath [12, 24-26]. Interestingly, in some dynamical systems, e.g. circuits with time delay and colored noise (see section 3.3), $\alpha$ can actually vary under changing operational conditions [27].

SDE (3) explicitly states the integration convention $\alpha^{\prime}$ and the noise-induced drift. As we have seen in section 1 , if desired, one can change the convention $\left(\alpha^{\prime}\right)$, but this entails a corresponding change in the drift coefficient $\left(\alpha-\alpha^{\prime}\right)$. For example,

$$
\mathrm{d} x_{t}=\underbrace{\sigma\left(x_{t}\right) \frac{\mathrm{d} \sigma\left(x_{t}\right)}{\mathrm{d} y}}_{\alpha-\alpha^{\prime}=1}+\underbrace{\sigma\left(x_{t}\right) \mathrm{d} W_{t}}_{\alpha^{\prime}=0},
$$

is equivalent to

$$
\mathrm{d} x_{t}=\underbrace{0.5 \sigma\left(x_{t}\right) \frac{\mathrm{d} \sigma\left(x_{t}\right)}{\mathrm{d} y}}_{\alpha-\alpha^{\prime}=0.5}+\underbrace{\sigma\left(x_{t}\right) \circ \mathrm{d} W_{t}}_{\alpha^{\prime}=0.5},
$$

and to

$$
\mathrm{d} x_{t}=\underbrace{\sigma\left(x_{t}\right) \circ_{1} \mathrm{~d} W_{t}}_{\alpha-\alpha^{\prime}=0, \alpha^{\prime}=1},
$$

where we are using the common notations $\sigma\left(x_{t}\right) \mathrm{d} W_{t} \equiv \sigma\left(x_{t}\right) \circ_{0} \mathrm{~d} W_{t}$ and $\sigma\left(x_{t}\right) \circ \mathrm{d} W_{t} \equiv \sigma\left(x_{t}\right) \circ_{0.5} \mathrm{~d} W_{t}$. In this review, unless otherwise stated, we will use the Itô convention $\left(\alpha^{\prime}=0\right)$ throughout and explicitly indicate the noiseinduced drifts to avoid misunderstandings associated with changing between different formalisms.

Before moving to the case studies in the next section, we want to make an important remark. In this section we have considered only first-order SDEs, where the presence of a noise-induced drift is related to the choice of a stochastic integration convention. In the case studies in section 3, we will typically start from a microscopic model of a system and eliminate some of its complexity to obtain an effective first-order SDE. The noise-induced drift present in the effective first-order SDE will, thus, be the result of this simplification process. For clarity, we will always write the effective SDEs using the Itô formalism, where the noise-induced drift is explicitly stated. For example, in sections 3.1 and 3.2, our starting point is a second-order equation, which we want to simplify further taking a parameter (e.g. mass of a particle) to zero. The resulting first-order Itô equation contains a drift term that combines the damping and the noise coefficients of the original equation. We emphasize that the source of this (physically measurable) additional drift is that we are taking a singular limit of a second-order equation in the presence of noise and we thus call it again a noise-induced drift. Its explicit form is now much harder to derive than in the case of the minimal model of section 2 . In the case discussed in section 3.1, it is possible (but not necessary) to interpret it in terms of a stochastic integration convention choice $(\alpha=1)$ $[28,29]$, as explained in section 2 , but no such interpretation is possible in the generality of the examples considered in section 3 .

\section{Case studies}

In section 2 we have seen how the presence of multiplicative noise induces a drift in a simple discrete-time model of a random walker. In the present section we consider in detail several examples of realistic models with a particular emphasis on those systems that have been subject of experiments. Section 3.1 considers Brownian motion of a microscopic particle in thermal equilibrium with a heat bath (i.e. for which the fluctuation-dissipation relations holds) in the presence of a diffusion gradient. Section 3.2 relaxes the condition that the system should be in equilibrium with a heat bath and thus considers systems for which a generalized fluctuation-dissipation relation holds. Section 3.3 considers the limiting behavior of a system driven by a colored noise modulated by a multiplicative delayed feedback. Finally, in section 3.4 we demonstrate how sensorial delay can alter the behavior of an autonomous agent in the presence of noise and how this effect can be used to control complex collective behaviors. In all cases we will present not only experimental findings, but also the basic mathematical methods and/or numerical techniques that can be employed to study a wide range of systems, while a more in-depth discussion of these methods and techniques is provided in the appendices.

\subsection{Brownian motion in a diffusion gradient}

Diffusion gradients emerge naturally when a Brownian particle is in a complex or crowded environment. For example, diffusion gets hindered when a particle is close to a wall due to hydrodynamic interactions: as shown in figure 2(c), the diffusion coefficient increases with the particle-wall distance approaching its bulk value at a distance of several particle radii away from the wall [9]. More generally, the study of diffusion in non-homogenous media is attracting a lot of attention in classical physical systems [30-35] as well as in biological systems [36] and in quantum systems [37]. The presence of a diffusion gradient introduces a multiplicative noise and thus leads to a noise-induced drift, often referred to in this context as a 'spurious drift'. The need to account for such spurious drifts was realized already several decades ago in the context of numerical simulations [19, 24, 38, 39], 
but only very recently did it become possible to observe them experimentally [12, 26, 40, 41].

In order to understand how spurious drifts emerge in the presence of diffusion gradients, we will consider a Brownian particle with mass $m$ moving in one dimension in a fluid at temperature $T$. Importantly, we assume that the particle is in thermal equilibrium with the heat bath provided by the fluid. The corresponding equation of motion is

$$
m \ddot{x}_{t}=F\left(x_{t}\right)-\gamma\left(x_{t}\right) \dot{x}_{t}+\gamma\left(x_{t}\right) \sqrt{2 D\left(x_{t}\right)} \eta_{t},
$$

where $F(x)$ denotes the sum of the external forces acting on the particle, $\gamma(x)$ is the position-dependent friction coefficient, $D(x)$ is the position-dependent diffusion coefficient, and $\eta_{t}$ is a unit white noise. Since we assume that the system is in thermal equilibrium, the intensity of the fluctuations $D(x)$ and the rate of energy dissipation $\gamma(x)$ satisfy the fluctuation-dissipation relation [42]

$$
D(x)=\frac{k_{\mathrm{B}} T}{\gamma(x)},
$$

where $k_{\mathrm{B}} T$ is the thermal energy and $k_{\mathrm{B}}$ is the Boltzmann constant. The equilibrium distribution of the system is given by the Boltzmann-Gibbs probability density

$$
\rho(x, v)=Z^{-1} \exp \left[-\frac{U(x)}{k_{\mathrm{B}} T}-\frac{m v^{2}}{2 k_{\mathrm{B}} T}\right],
$$

where $U(x)$ is the potential of the (external) forces $F(x)$ so that $F(x)=-\frac{\mathrm{d} U(x)}{\mathrm{d} x}$, and we are assuming that the density is normalizable with $Z$ denoting the normalizing factor. Furthermore, the Maxwellian velocity distribution $\left(\propto \exp \left[-\frac{m v^{2}}{2 k_{\mathrm{B}} T}\right]\right)$ implies energy equipartition, so that the equilibrium kinetic energy is on average equal to the thermal energy:

$$
\left\langle\frac{1}{2} m v_{t}^{2}\right\rangle=\frac{1}{2} k_{\mathrm{B}} T
$$

In SDE (14), inertial effects decay on a very short time scale, i.e. the momentum relaxation time $\tau_{\mathrm{m}}=m / \gamma$, which is typically of the order of a fraction of a microsecond ${ }^{5}$. For example, for a silica microsphere with radius $R=1 \mu \mathrm{m}$ $(m=11 \mathrm{pg})$ in water at room temperature $(T=300 \mathrm{~K})$, $\tau_{\mathrm{m}}=0.6 \mu \mathrm{s}$. This time is several orders of magnitude shorter than the time scales of typical experiments, which are of the order of milliseconds or longer 6 . Thus, it is justified to take the limit $m \rightarrow 0$ in SDE (14). This has to be done carefully and requires a nontrivial calculation. In particular, it is not correct to simply set $m=0$ and drop the inertial term. As a result of the calculation outlined in appendix B, we obtain the effective SDE

$\mathrm{d} x_{t}=\frac{F\left(x_{t}\right) D\left(x_{t}\right)}{k_{\mathrm{B}} T} \mathrm{~d} t \underbrace{+\frac{\mathrm{d} D\left(x_{t}\right)}{\mathrm{d} x} \mathrm{~d} t}_{\text {spurious drift }}+\sqrt{2 D\left(x_{t}\right)} \mathrm{d} W_{t}$.

${ }^{5}$ In a liquid environment, furthermore, also the hydrodynamic memory of the fluid, i.e. the mass of the fluid displaced together with the particle, must be taken into account and can, in fact, significantly increase the effective momentum relaxation time $[88,89]$.

${ }^{6}$ In fact, recent experiments have been able to resolve the inertial regime of Brownian particles immersed both in a gas and in a liquid. For a recent review see [43].
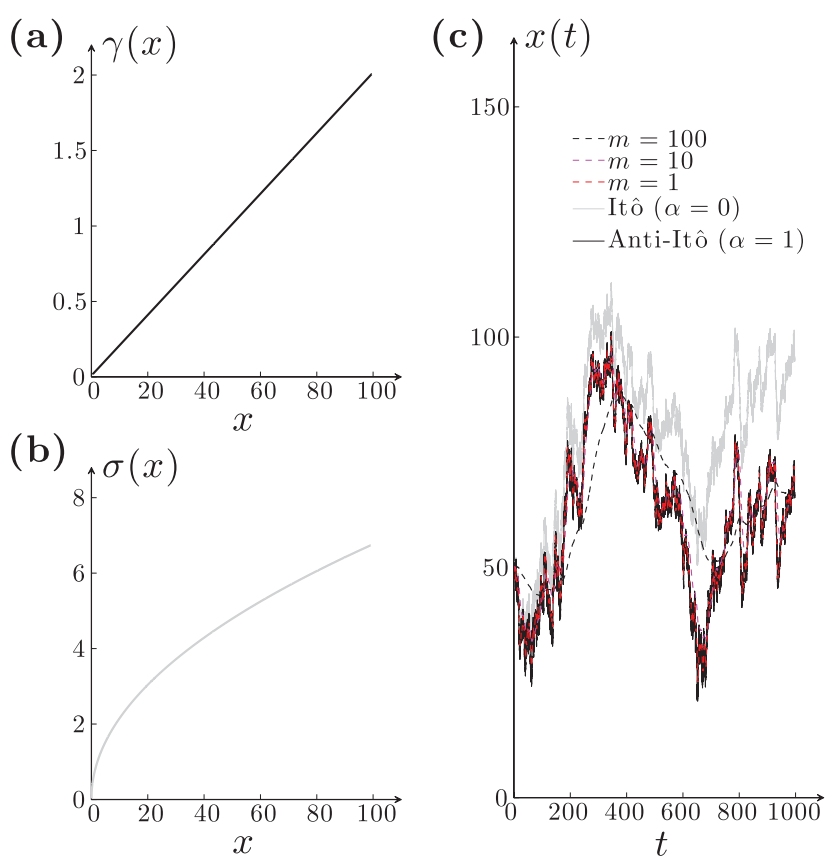

Figure 5. Limiting SDE for a system satisfying the fluctuationdissipation relation. For a Brownian particle in thermal equilibrium in a diffusion gradient (a) $\gamma(x)$ and (b) $\sigma(x)$ are related by the Einstein fluctuation-dissipation relation (equation (15)). (c) The solutions of the equations of motion (SDE (14)) for $m \rightarrow 0$ (dashed lines) converge to the solution of the limiting SDE (18), including the spurious drift (black solid line). The (physically incorrect) solution without spurious drift (grey solid line) is given for comparison. All solutions are numerically calculated using the same realization of the Wiener process, with $F(x) \equiv 0$.

Note that, if we denote the noise coefficient $\sqrt{2 D(x)}$ by $\sigma(x)$ the noise-induced drift equals $\frac{\mathrm{d} D(x)}{\mathrm{d} x}=\sigma(x) \frac{\mathrm{d} \sigma(x)}{\mathrm{d} x}$.

The numerical simulations shown in figure 5 give us some insight into the derivation of the limiting SDE and the emergence of the noise-induced drift. We simulate a Brownian particle at equilibrium with a thermal bath, so that the coefficients $\gamma(x)$ (figure 5(a)) and $D(x)$ (figure 5(b)) are related by the Einstein fluctuation-dissipation relation (equation (15)). The dashed lines in figure 5(c) represent solutions of SDE (14) obtained for decreasing values of $m$, but with the same realization of the driving Wiener process. These solutions become rougher as $m$ decreases and converge towards the solution of the limiting SDE (18) (black solid line in figure 5(c)), again calculated using the same realization of the Wiener process. We see that omitting the spurious drift leads to clear deviations, which diverge as a function of time (grey solid line in figure 5(c)).

The noise-induced drift in SDE (18) has been directly observed in at least two sets of experiments. Before proceeding further, we note that, in general, the diffusion $D(x)$ and (total) drift $C(x)$ of an experimental system can be obtained from an experimental discrete time-series $\left\{x_{1}, \ldots, x_{N}\right\}$ sampling the system's state at regular intervals $\Delta t$ as the conditional averages

$$
D(x)=\frac{1}{2 \Delta t}\left\langle\left(x_{n+1}-x_{n}\right)^{2} \mid x_{n} \cong x\right\rangle
$$


and

$$
C(x)=\frac{1}{\Delta t}\left\langle x_{n+1}-x_{n} \mid x_{n} \cong x\right\rangle .
$$

In experiments, $\Delta t$ should be much smaller than the characteristic relaxation time of the system, which is determined by the drift part of the SDE and is typically several orders of magnitude larger than $\tau_{\mathrm{m}}$. Furthermore, in the limit $\Delta t \rightarrow 0$ inertial effects come into play [43] and, therefore, in practice equations (19) and (20) should only be used in the overdamped limit, i.e. for $\Delta t \gg \tau_{\mathrm{m}}$. Similar considerations hold also for other microscopic dynamics determining the evolution of the system, i.e. $\Delta t$ should be much longer than the characteristic times of the dynamics that have been homogenized in the effective SDE.

The first direct experimental observation of the noiseinduced drift was performed by Lançon et al [12] who studied the Brownian motion of particles confined between two nearly parallel walls. The experimental sample was realized by placing a droplet of colloidal suspension between a spherical lens (with curvature $L$ ) and a flat disk, as shown in figure 6(a). The spacing $h$ between the flat and curved walls depended on the distance $r$ from the center of the cell as $h \approx r^{2} /(2 L)$. The colloidal solution consisted of polystyrene spheres (radius $R=1$ $\mu \mathrm{m}$ ) suspended in a mixture of $\mathrm{H}_{2} \mathrm{O}$ and $\mathrm{D}_{2} \mathrm{O}$ adjusted to cancel any sedimentation effects. The horizontal Brownian motion of the particles was observed using digital video microscopy. The experimental values of the ratio between the measured diffusion coefficient parallel to the walls $D_{\|}(h)$ and the bulk diffusion coefficient $D_{\mathrm{SE}}$ were inferred from the measured trajectories using equation (19) and are shown in figure 6(b) (white squares). For the measurement of the noise-induced drift, the center of the observation frame was fixed at a position with $y=0$ and $x=300 \mu \mathrm{m}$ (inset in figure 6(a)), corresponding to an average relative confinement $h /(2 R)=1.5$ so that all particles present in the frame were outside of the excluded volume (i.e. $h \leqslant 2 R$ ) and had a diffusion coefficient with the largest $x$-dependence, but no $y$-dependence (to first order). The drift of the Brownian particles over a period of about three minutes is shown in figure 6(c). Importantly, no flux and no concentration gradient were observed over a period of a week or more, which is consistent with the (uniform) Boltzmann distribution expected in the absence of external forces and in thermal equilibrium (equation (16)).

In [26] and [41], we studied the Brownian motion of a colloidal particle in water with a diffusion gradient imposed by the presence of the bottom wall of the sample cell, as shown in figure 7(a). The external forces acting on the particle were gravity and electrostatic repulsion from the bottom of the sample cell. Since both are vertical, one can separate the horizontal degrees of freedom and write the equation of motion for the vertical coordinate only, which we will call $z . D_{\perp}(z)$ decreases near the bottom of the sample cell and its precise form can be found in [9] (see also figure 2(c)). The trajectory of a particle close to the wall was measured with total internal reflection microscopy (TIRM), which is a technique that permits one to measure the position of a colloidal particle above a surface with nanometer resolution [44]. From the measured trajectories we obtained (a)

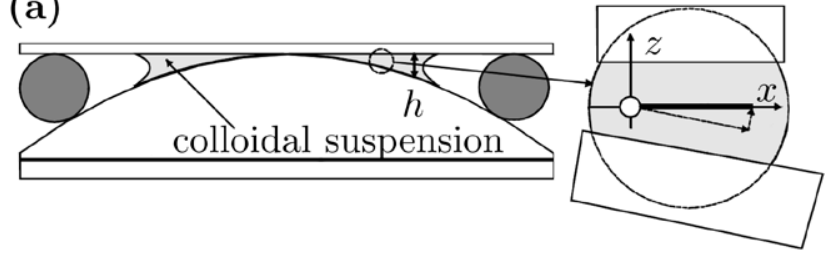

(b)

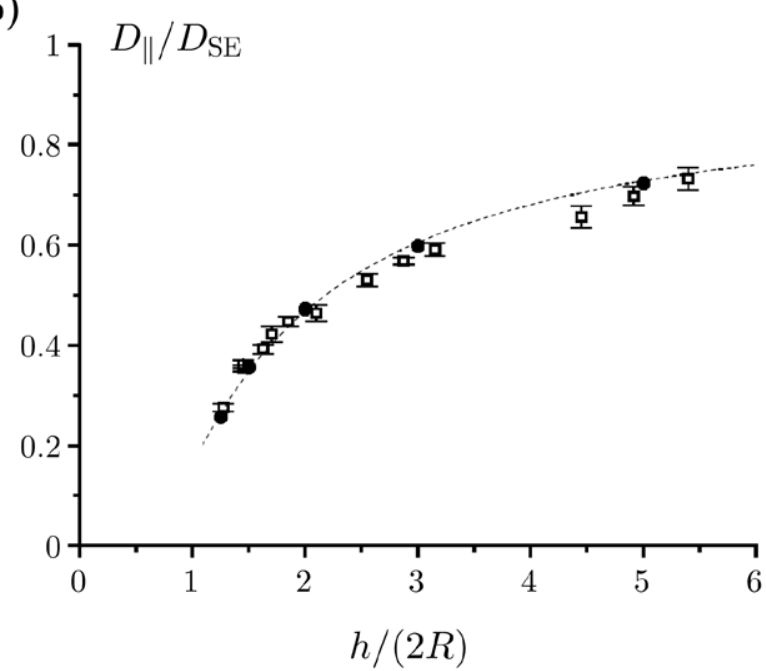

(c)

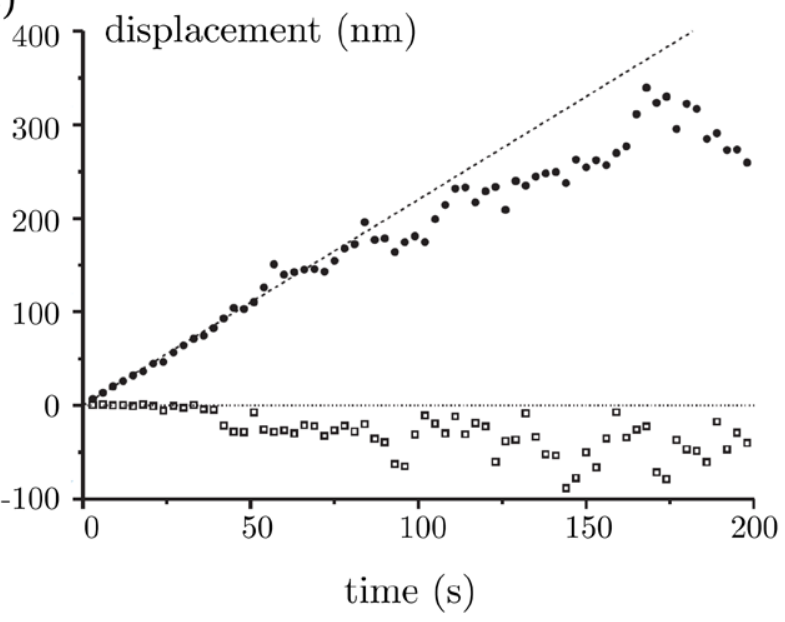

Figure 6. Drift without flux. (a) Cross-section of a sample cell where a colloidal suspension (particle radius $R=1 \mu \mathrm{m}$ ) is confined between a spherical lens and a flat disk, separated by an elastic O-ring. The round inset identifies the observation frame. The height of the cell is denoted by $h$. (b) Diffusion coefficient parallel to the walls $D_{\|}$ normalized to the bulk diffusion coefficient $D_{\mathrm{SE}}$ as a function of the relative confinement $h /(2 R)$. The open squares are the experimental data; the dotted line is the best fit to the black dots, which correspond to numerical predictions calculated by the collocation method. (c) Drift of the walkers as a function of time along the diffusion gradient (black dots) and perpendicular to the diffusion gradient (open squares). Adapted with permission from [12]. Copyright 2001 EDP Sciences.

$D_{\perp}(z)$ using equation (19) (symbols in figure 7(b)), which is in a very good agreement with the theoretical prediction [9] (line in figure 7(b)). We were then able to directly measure the spurious drift for particles of various sizes, as shown in figure 7(c).

We conclude this section with a brief discussion of how the presence of a noise-induced drift plays a crucial role in 

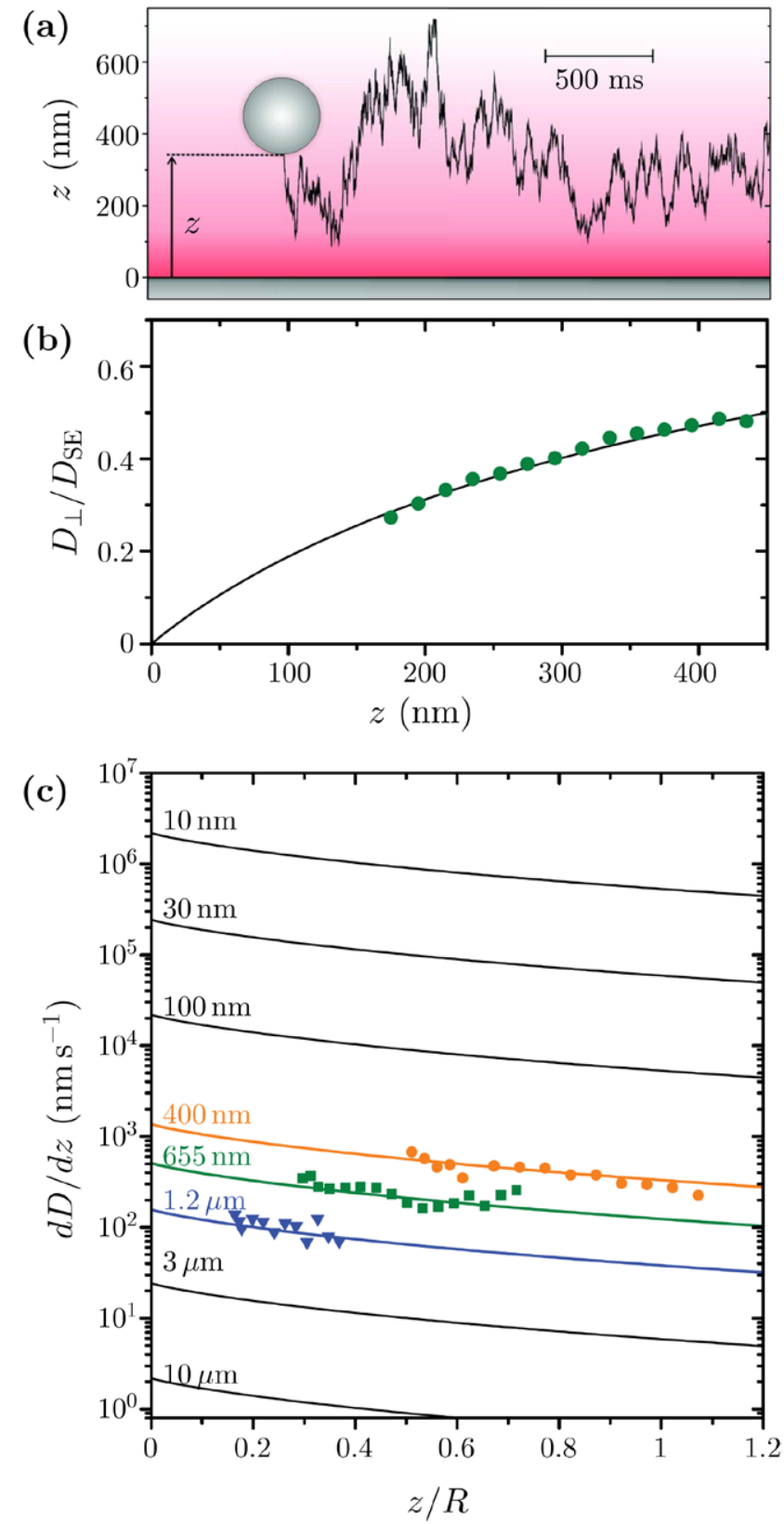

Figure 7. Experimental measurement of spurious drifts. (a) A Brownian particle (drawn not to scale) diffuses above a wall in the presence of gravitational and electrostatic forces. Its trajectory's component in the direction perpendicular to the wall is measured with total internal reflection microscopy (TIRM). Adapted with permission from [26]. Copyright 2010 American Physical Society. (b) Comparison of measured (symbols) and calculated (line) normalized vertical diffusion coefficient $D_{\perp}(z) / D_{\mathrm{SE}}$ for an $R=400 \mathrm{~nm}$ particle as a function of the particle-wall separation $z$. (c) Distance dependence of the theoretically calculated spurious drift $\frac{\mathrm{d} D(z)}{\mathrm{d} z}$ for various particle radii $R$ (lines). Experimentally measured spurious drifts are shown for $R=400 \mathrm{~nm}$ (circles), $R=655 \mathrm{~nm}$ (squares) and $R=1180 \mathrm{~nm}$ (triangles). Adapted with permission from [41]. Copyright 2011 American Physical Society.

the measurement of forces acting on Brownian particles in a liquid medium. The forces acting on a microscopic object immersed in a liquid medium can be assessed either by their underlying potential or by their effect on the object's trajectory $[26,41]$. The first approach-to which we shall refer as equilibrium distribution method-requires sampling of the equilibrium distribution $\rho(x)$ of the particle's position (see also equation (16)). The force can then be obtained from

$$
F(x)=-\frac{\mathrm{d} U(x)}{\mathrm{d} x}=\frac{k_{\mathrm{B}} T}{\rho(x)} \frac{\mathrm{d} \rho(x)}{\mathrm{d} x} .
$$

This method can only be applied under conditions where the investigated system is in thermodynamic equilibrium with a heat bath. The second method-to which we shall refer as drift method-does not require the object to be in (or even close to) thermal equilibrium. This method requires obtaining $D(x)$ and $C(x)$ from experimental trajectories (equations (19) and (20)) and including a correction for the presence of a spurious (noise-induced) force. The force can then be calculated as

$$
F(x)=\gamma(x) C(x) \underbrace{-\gamma(x) \frac{\mathrm{d} D(x)}{\mathrm{d} x}}_{\text {spurious force }} .
$$

This method has the advantage that it can be applied also to systems that are intrinsically out-of-equilibrium, e.g. molecular machines, transport through pores, DNA stretching; however, it requires recording the object's trajectory with high sampling rates, which can be technologically challenging, in particular when combined with a high spatial resolution. For example, the correction due to the presence of spurious forces has been taken into account in the experimental simultaneous determination of potential and diffusivity landscapes in macromolecular solutions [45].

\subsection{Diffusive systems not satisfying the fluctuation- dissipation relation}

While in section 3.1 we considered systems in thermal equilibrium with a heat bath that satisfy the fluctuation-dissipation relation (equation (15)), in this section we consider the zeromass limiting behavior of a larger class of models for which $\gamma(x)$ and $\sigma(x)$ are allowed to vary independently from each other. This is a very general class of noisy dynamical systems, with many interesting examples and applications (see, e.g. Ao et al [46] and [47]). Using the methods of [48], we will thus study the general SDE

$$
m \ddot{x}_{t}=F\left(x_{t}\right)-\gamma\left(x_{t}\right) \dot{x}_{t}+\sigma\left(x_{t}\right) \eta_{t},
$$

where the damping and diffusion terms are not necessarily related by the fluctuation-dissipation relation (equation (15)). For a wide class of such systems the effective equation in the $m \rightarrow 0$ limit is

$\mathrm{d} x_{t}=[\frac{F\left(x_{t}\right)}{\gamma\left(x_{t}\right)} \underbrace{-\frac{\sigma\left(x_{t}\right)^{2}}{2 \gamma\left(x_{t}\right)^{3}} \frac{\mathrm{d} \gamma\left(x_{t}\right)}{\mathrm{d} x}}_{\text {noise-induced drift }}] \mathrm{d} t+\frac{\sigma\left(x_{t}\right)}{\gamma\left(x_{t}\right)} \mathrm{d} W_{t}$.

An outline of the mathematical derivation of this equation is provided in appendix C. An example of such a system is illustrated in figure 8: for the case with $\gamma(x) \equiv$ constant (figure $8(\mathrm{a})$ ) and $\sigma(x)$ state-dependent (figure $8(\mathrm{~b})$ ), the 


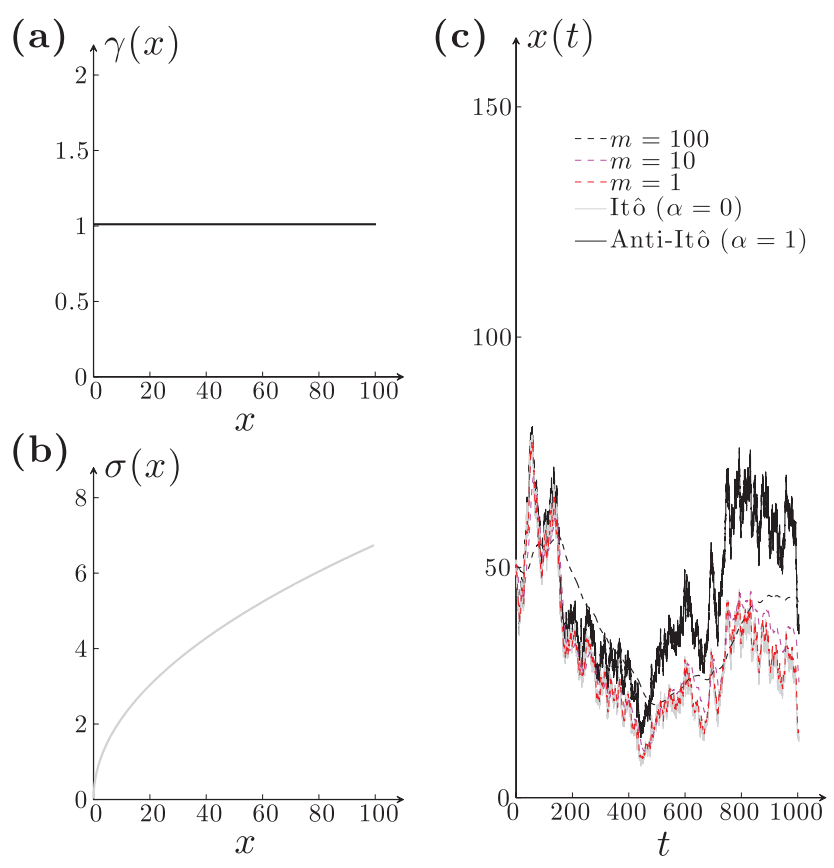

Figure 8. Limiting SDE for a system not satisfying the fluctuationdissipation relation. Consider a system for which (a) $\gamma(x) \equiv$ constant and (b) $\sigma(x)$ is state-dependent. (c) The solutions of $\operatorname{SDE}(23)$ for $m \rightarrow 0$ (dashed lines) converge to the solution of the approximate SDE (24), which in this case corresponds to the Itô interpretation (grey solid line) of the equation $\mathrm{d} x_{t}=\frac{F\left(x_{t}\right)}{\gamma} \mathrm{d} t+\frac{\sigma\left(x_{t}\right)}{\gamma} \mathrm{d} W_{t}$, as the noise-induced drift equals zero $(\alpha=0)$ when $\gamma$ is constant. The solution for the anti-Itô integral ( $\alpha=1$, black solid line) is given for comparison. All solutions are obtained for the same realization of the Wiener process.

solutions of SDE (23) for $m \rightarrow 0$ (dashed lines in figure 8(c)) converge to the solution of the approximate SDE (24) (grey solid line in figure 8(c)); note that in this case the noiseinduced drift is zero, differently from the system considered in section 3.1.

In general, there is no relation between noise and damping coefficients if the noise is external, as in an electrical circuit driven by a noise source. Such a circuit with a colored noise and involving a delayed response is studied in section 3.3. Another physical example described by an equation of the form of SDE (23) is diffusion of a Brownian particle in a temperature gradient. This system shows more interesting phenomena when it is driven by a colored noise; a simple model of this type is studied in [49]. Brownian motion in a diffusion gradient, discussed in section 3.1, is yet another special case of a system described by SDE (23) and the result outlined there is a special case of SDE (24).

SDE (23) can be generalized to multidimensional (i.e. vector) systems as

$\left\{\begin{array}{l}\mathrm{d} \boldsymbol{x}_{t}^{m}=\boldsymbol{v}_{t}^{m} \mathrm{~d} t, \\ \mathrm{~d} \boldsymbol{v}_{t}^{m}=\left[\frac{\boldsymbol{F}\left(\boldsymbol{x}_{t}^{m}\right)}{m}-\frac{\gamma\left(\boldsymbol{x}_{t}^{m}\right)}{m} \boldsymbol{v}_{t}^{m}\right] \mathrm{d} t+\frac{\boldsymbol{\sigma}\left(\boldsymbol{x}_{t}^{m}\right)}{m} \mathrm{~d} \boldsymbol{W}_{t},\end{array}\right.$

where $\mathbf{W}$ is a vector Wiener process (i.e. the components of $\mathbf{W}$ are independent Wiener processes), and $\gamma$ and $\boldsymbol{\sigma}$ are matrices. The form of the limiting equation is again: $\mathrm{d} \boldsymbol{x}_{t}=\left[\gamma^{-1}\left(\boldsymbol{x}_{t}\right) \boldsymbol{F}\left(\boldsymbol{x}_{t}\right)+\boldsymbol{S}\left(\boldsymbol{x}_{t}\right)\right] \mathrm{d} t+\gamma^{-1}\left(\boldsymbol{x}_{t}\right) \boldsymbol{\sigma}\left(\boldsymbol{x}_{t}\right) \mathrm{d} \boldsymbol{W}_{t}$,

The precise expression for $\mathbf{S}$ and a sketch of its derivation are provided in appendix C. The zero-mass limits of equations similar to SDE (25) have been studied by many authors beginning with Smoluchowski [50] and Kramers [51]. In the case where $F=0$ and $\gamma$ and $\sigma$ are constant, the solution to SDE (23) converges to the solution of SDE (24) almost surely [2]. Schuss [52] treated the case including an external force by entirely different methods. Hänggi [19] identified the limit with position-dependent noise and friction for the case when the fluctuation-dissipation relation is satisfied and Sancho et al [39] for the general one-dimensional case (the multidimensional case is also discussed there but without complete proof). Hottovy et al [47] used the homogenization techniques described in [53, 52] and [54] to compute the limiting backward Kolmogorov equation corresponding to equation (23) as mass is taken to zero. Pardoux and Veretennikov [55] proved rigorously convergence in distribution for equations of the same type as SDE (25), under somewhat stronger assumptions than those made in [48]. Freidlin [56] gave the first rigorous proof of strong convergence in the zero-mass limit for $\gamma$ constant and $\boldsymbol{\sigma}$ position-dependent. Hottovy et al [48] provided the first rigorous derivation of the zero-mass limit of SDE (25) for a multidimensional system with general friction and noise coefficients.

The general form of SDE (25) allows to treat many interesting physical situations, including the case when the force $\mathbf{F}$ is not conservative. In this case, there is no known explicit formula for the stationary measure of the dynamics defined by SDE (25), even when the system satisfies the fluctuationdissipation relation. Nevertheless, the general theorem applies, giving the limiting equation for $x_{t}$.

As another application of the general scheme given by SDE (25), suppose the white noise in SDE (23) is replaced by a colored (i.e. time-correlated) stationary noise process, which is itself a solution of a stochastic differential system. For example, $\eta^{\tau}$ may be an Ornstein-Uhlenbeck process:

$$
\mathrm{d} \eta_{t}^{\tau}=-\frac{a}{\tau} \eta_{t}^{\tau} \mathrm{d} t+\frac{1}{\tau} \mathrm{d} W_{t}
$$

Defining $\chi_{t}=\int_{0}^{t} \eta_{s}^{\tau} \mathrm{d} s$, we introduce a new, compound space variable $(x, \chi)$ and the corresponding velocity $\left(v, \eta^{\tau}\right)$. If the parameter $\tau$ scales linearly with $m$, the variables $(x, \chi)$ and $\left(v, \eta^{\tau}\right)$ satisfy a system of equations of the same form as SDE (25). The above general result applies, yielding an effective equation for a system in which the momentum relaxation time $\tau_{\mathrm{m}}$ and the characteristic noise correlation time $\tau$ go to zero at the same rate. The details are given in [48]. In section 3.3 we will see that $\tau$ can also interact with the feedback delay time of the system.

The well-known Itô-to-Stratonovich correction [5] can be presented (using Wong-Zakai theorem [57]) as a special case of noise-induced drift. However, the latter is much more general and can be nonzero even when the noise coefficient is constant. 


\subsection{Delayed multiplicative feedback and colored noise}

White noise does not exist in real systems, since its correlation time is strictly equal to zero (and even as a mathematical object it does not have well-defined realizations which would be functions of time) [5]. Colored noises are more regular mathematical objects, and more similar to signals that can be actually generated. Thus, it is natural to consider SDEs driven by colored noise. An SDE with colored noise can be interpreted as a usual ordinary differential equation for each noise realization. However, the very correlation effects we want to model make such equations harder to study. The characteristic time of the noise correlations, $\tau$, becomes an important time scale of the model, whose properties often simplify in the limit when $\tau \rightarrow 0$. Such limit is studied in the classic work of Wong and Zakai [57], who considered a sequence of SDEs driven by colored noises with symmetric covariance functions and with correlation times $\tau_{n} \rightarrow 0$ and showed that their solutions converge to the solution of the corresponding Stratonovich equation driven by the white noise. A more general result can be found in [58]; see also [59], where such limits are studied using homogenization methods, and [60]. We remark that all these results can be recovered by the methods discussed in section 3.2 and in appendix $\mathrm{C}$.

A system obeying an SDE with a colored noise was experimentally realized by Smythe et al [22] as an eletrical circuit driven by a multiplicative noisy voltage input. Depending on the mean and variance of the noise, the output voltage of the circuit could have a probability density with either one or two maxima, and the precise form of the phase diagram depended on whether the equation describing the circuit was interpreted using Itô or Stratonovich integral. As shown in figure 9, the results of [22] were in quite good agreement with the theoretical predictions based on the Stratonovich interpretation, illustrating the role of the colored noise, as mathematically described by the Wong-Zakai theorem.

We will now consider in detail the experiment performed by Pesce et al [27] using an RC electric circuit driven by a multiplicative colored noise (figure 10(a)), in which the output voltage was fed back into the system and multiplicatively coupled to the noise source, after going through a nonlinear filter. Unlike the circuit studied by Smythe et al [22], the circuit studied by Pesce et al [27] involved a delay in the feedback cycle. The SDE describing the evolution of the voltage in the circuit presented in figure 10(a) is

$$
\mathrm{d} x_{t}=-k x_{t} \mathrm{~d} t+\sigma F\left(x_{t-\delta}\right) \eta_{t}^{\tau} \mathrm{d} t,
$$

where $k=(R C)^{-1}, R$ is the resistance of the circuit, $C$ is its capacitance, and $F(x)$ represents the modulation by the filter. The colored noise $\eta^{\tau}$ is an Ornstein-Uhlenbeck process with mean zero and with the characteristic time of correlation decay equal to $\tau$ (i.e. the stationary solution of SDE (27) with $a=1) . \delta$ is the time delay resulting from the application of the filter and $\sigma$ denotes the (constant) noise intensity.

We studied SDE (28) in the limit of small $\tau$ and $\delta$. Mathematically, this meant making $\tau$ and $\delta$ proportional to a small parameter $\varepsilon$ and taking the limit $\epsilon \rightarrow 0$, keeping the ratio $\delta / \tau$ constant. The limiting SDE turned out to be

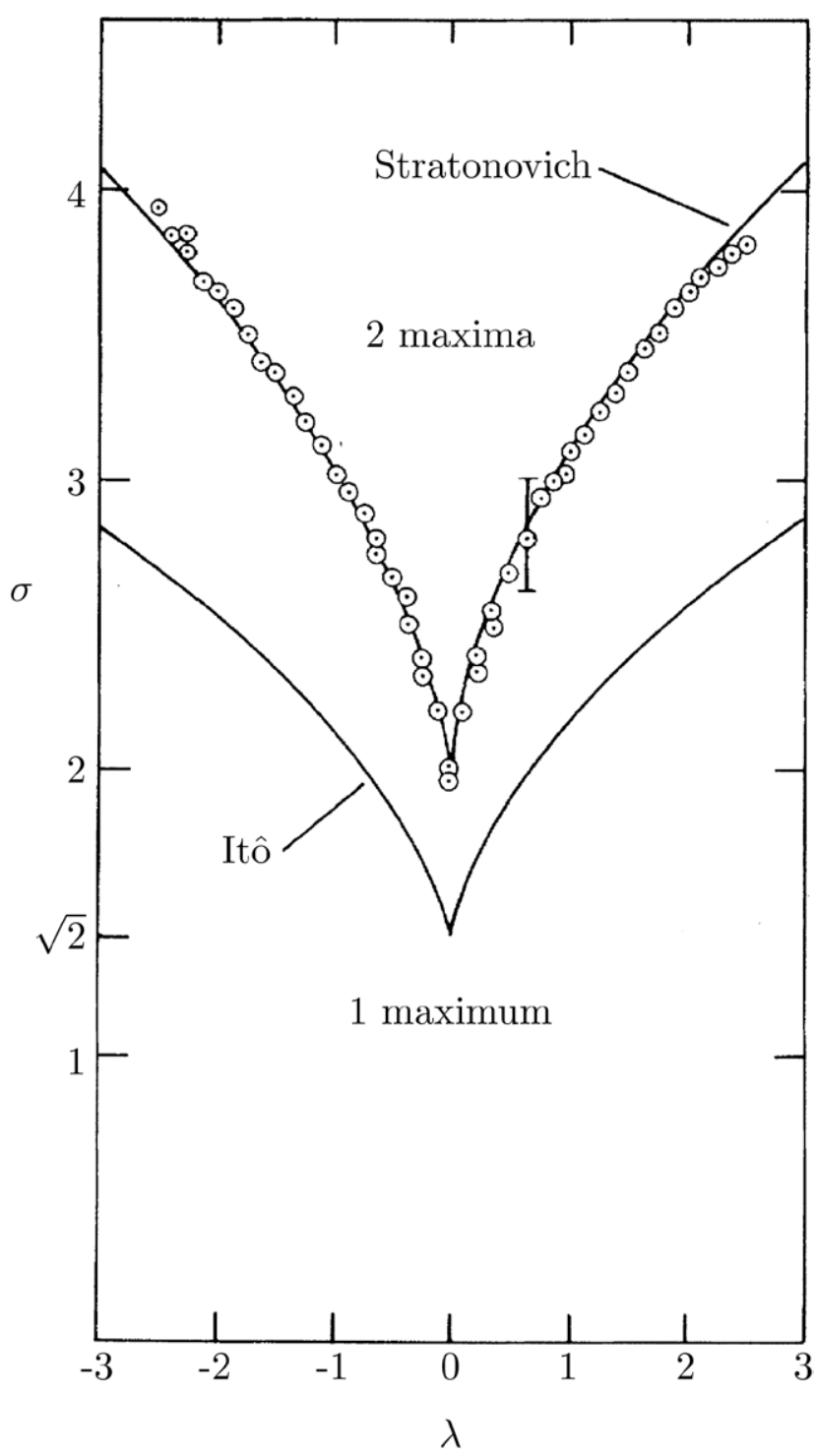

Figure 9. Experimental observation of a noise-induced phase transition. Phase diagram of the electric circuit with multiplicative noise used by Smythe et al [22]. Depending on the input noise parameters, namely its mean $\lambda$ and its standard deviation $\sigma$, the circuit could be either monostable ( 1 maximum) or bistable ( 2 maxima). The experimentally measured transition between the two regimes (open circles) agrees with the predictions obtained by integrating the SDE describing the circuit according to the Stratonovich convention. The reason for this is that the driving noise is colored. The predictions according to the Itô convention are shown for comparison. Adapted with permission from [22]. Copyright 1983 American Physical Society.

$\mathrm{d} x_{t}=[-k x_{t} \underbrace{+\alpha \sigma^{2} F\left(x_{t}\right) \frac{\mathrm{d} F\left(x_{t}\right)}{\mathrm{d} x}}_{\text {noise-induced drift }}] \mathrm{d} t+\sigma F\left(x_{t}\right) \mathrm{d} W_{t}$.

The outline of the derivation is provided in appendix $\mathrm{D}$. The second term has the same structure as the noise-induced drift in the Brownian motion case: it is proportional to the product of the original noise coefficient, $\sigma F(x)$, and its spatial derivative (see section 3.1). The proportionality constant depends on the time scales of the problem as 
(a)

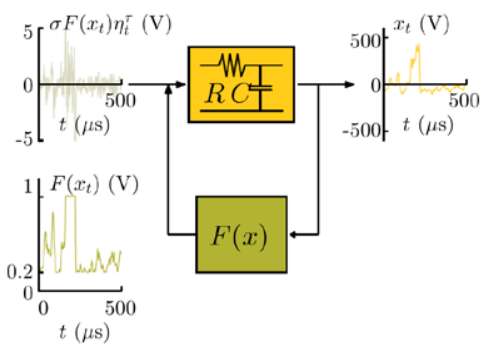

(c)

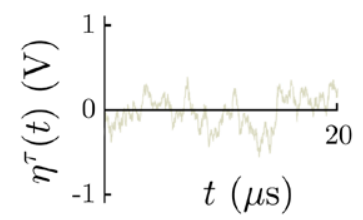

(b)

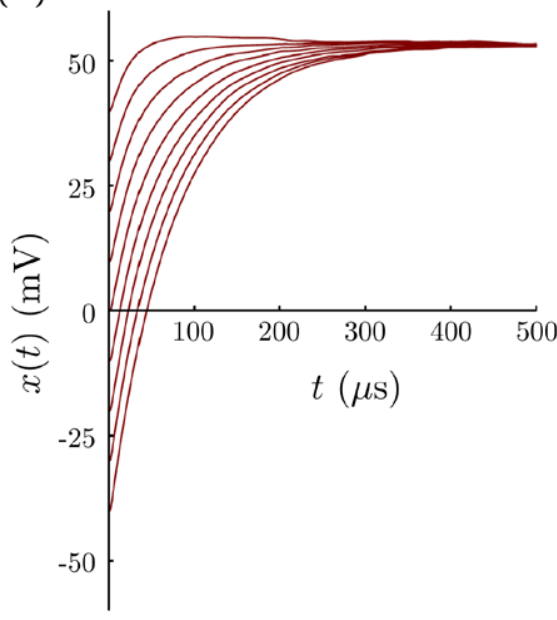

(d)
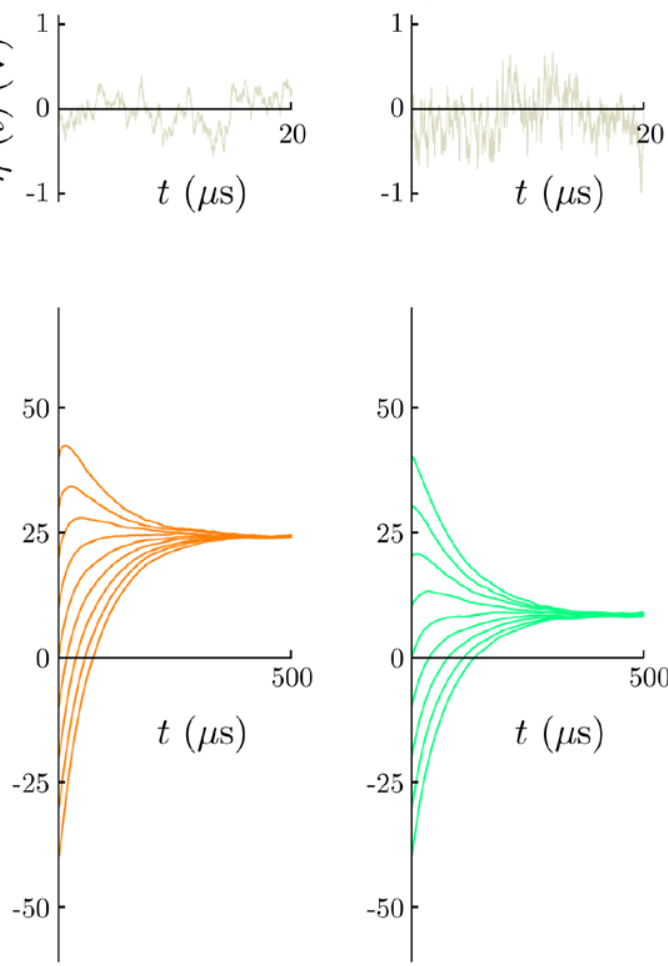

(e)
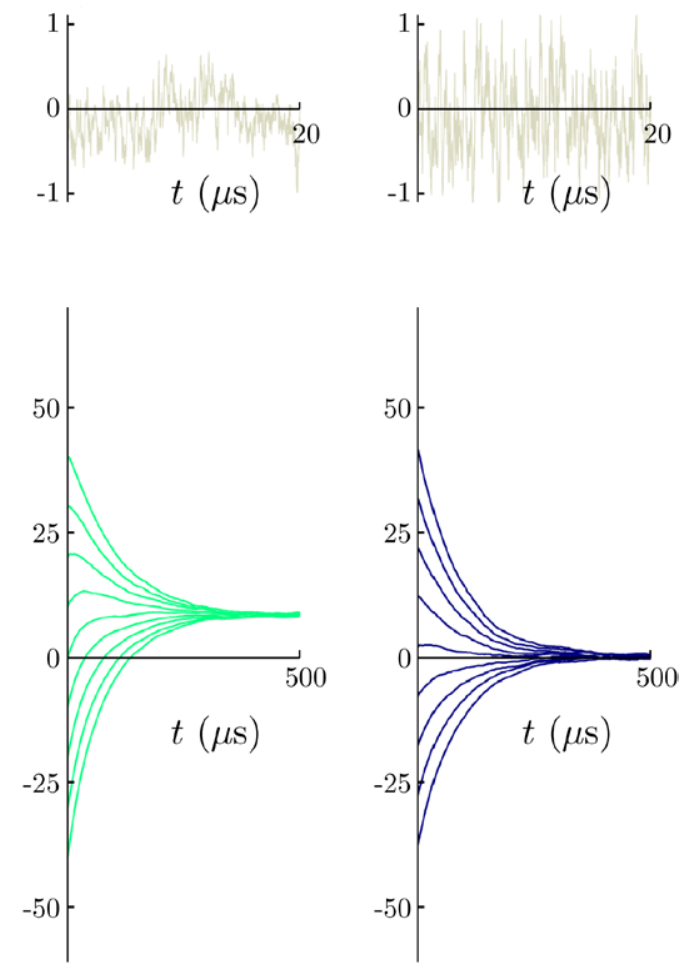

Figure 10. Stochastic dynamical system driven by multiplicative noise with delayed feedback. (a) Schematic representation of a stochastic dynamical system (an electric circuit) with multiplicative feedback $F(x)$ : the driving colored noise $\eta_{t}^{\tau}(\tau=1.1 \mathrm{~ms})$ is multiplied by a function of the system's state $x_{t}$. (b) Average of 1000 trajectories for various initial conditions. These results are in agreement with the Stratonovich treatment of the circuit SDE. ((c)-(e)) Samples of input noises $\eta_{t}^{\tau}$ (top) and average of 1000 trajectories (bottom) for various initial conditions with $\tau=0.6,0.2$ and $0.1 \mathrm{~ms}$ respectively for (c)-(e). From (b)-(e), one sees a shift of the equilibrium towards $x=0$, corresponding to a crossover from the Stratonovich solution to the Itô solution of the circuit SDE. Adapted with permission from [27]. Copyright 2013 Nature Publishing Group.

$$
\alpha=\frac{0.5}{1+\frac{\delta}{\tau}},
$$

which agrees well with the experimental results, as shown in figure 11; see also [90] where a more precise calculation yields $\alpha=\frac{1}{2} \mathrm{e}^{-\frac{\delta}{T}}$. SDE (29) is written here in the Itô form, but it can be interpreted according to another convention, corresponding to another choice of the parameter $\alpha$, as described in section 2. In this language, the presence of such delay made the SDE describing the behavior of the electric circuit with multiplicative noise cross over from obeying the Stratonovich convention $(\alpha=0.5)$ to obeying the Itô convention $(\alpha=0)$, as the ratio between the colored noise correlation time $\tau$ and the feedback delay $\delta$ varied (equation (30)), as shown in figures 10(b)-(e). The fact that this transition occurs as $\tau$ becomes close to $\delta$, i.e. $\delta / \tau \approx 1$ (figure 11 ), can be qualitatively explained as follows: if $\delta=0$, there is a correlation between the sign of the input noise and the time derivative of the feedback signal (which is the underlying reason why the process converges to the Stratonovich solution [57]); however, if $\delta \gg \tau$, this correlation disappears, effectively randomizing the time-derivative of the feedback signal with respect to the sign of the input noise and leading to a situation where the system loses its memory. While this crossover between two stochastic integration conventions was emphasized in [27], we

remark here that this is just a possible way of interpreting the noise-induced drift.

McDaniel et al [61] study theoretically a much more general system of delayed SDEs driven by several colored noises with couplings that are functions of the delayed dynamical variables. The corresponding results also are outlined in appendix D. A more accurate results was recently derived by Hottovy et al [90].

\subsection{Delayed sensorial feedback}

Another example in which the effective behavior of a diffusive system depends on a relation between two characteristic time scales occurs in the motion of autonomous agents, such as robots [62] and active Brownian particles [63, 64], whose speed depends on position and whose velocity's direction changes randomly. Mijalkov et al [62] performed an experimental, theoretical, and numerical study of such a system in two and (for theory and numerics) also in three dimensions. For simplicity, we discuss here only the planar case.

In the first part of the experiment performed by Mijalkov et al [62], a single robot moves in a gradient of light intensity. The robot reacts to the local light intensity by adjusting its speed, possibly with a time shift (positive or negative, as explained below). The robot's orientation (and, thus, the direction of 


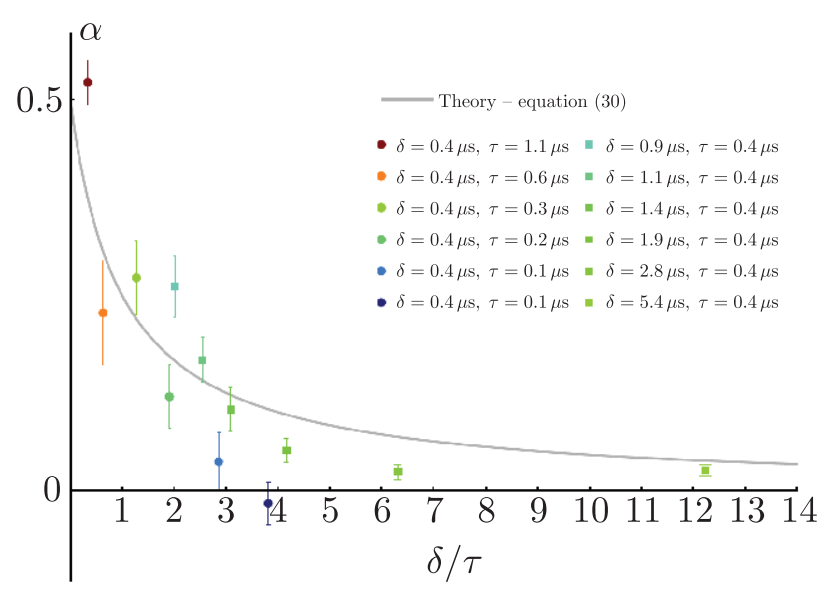

Figure 11. Dependence of $\alpha$ on $\delta / \tau$. $\alpha$ varies from 0.5 (Stratonovich integral) to 0 (Itô integral) as $\delta / \tau$ increases. The solid line represents the theoretical results (equation (30)); the dots represent the experimental values of $\alpha$ for fixed $\delta=0.4 \mu$ s and varying $\tau$; and the squares the experimental values for fixed $\tau=0.4 \mu \mathrm{s}$ and varying $\delta$. The error bars represent one standard deviation obtained by repeating the experimental determination of the ratio $\delta / \tau$ ten times. Adapted with permission from [27]. Copyright 2013 Nature Publishing Group.

its velocity) changes randomly in time. The robot's motion is studied in the regime where the time shift is small and the orientation changes occur fast. The idealized SDEs describing the robot's motion are thus

$$
\left\{\begin{aligned}
\mathrm{d} x_{t} & =\frac{1}{\sqrt{\tau}} u\left(x_{t-\delta}, y_{t-\delta}\right) \cos \phi_{t} \mathrm{~d} t \\
\mathrm{~d} y_{t} & =\frac{1}{\sqrt{\tau}} u\left(x_{t-\delta}, y_{t-\delta}\right) \sin \phi_{t} \mathrm{~d} t \\
\mathrm{~d} \phi_{t} & =\sqrt{\frac{2}{\tau}} \mathrm{d} W_{t}
\end{aligned}\right.
$$

where $\left(x_{t}, y_{t}\right)$ represents the position of the robot, $\phi_{t}$ is its orientation, $u\left(x_{t-\delta}, y_{t-\delta}\right)$ is the speed of the robot, which is related to the local light intensity measured by the robot with a delay $\delta$, and $\tau$ is the characteristic time with which the robot's orientation changes. In order to realize the model experimentally and to perform numerical simulations, we linearized the first two SDEs (31), obtaining

$\left\{\begin{array}{l}\dot{x}_{t}=\frac{1}{\tau}\left[u\left(x_{t}, y_{t}\right)-\delta \frac{\partial u}{\partial x}\left(x_{t}, y_{t}\right) \dot{x}_{t}-\delta \frac{\partial u}{\partial y}\left(x_{t}, y_{t}\right) \dot{y}_{t}\right] \cos \phi_{t} \\ \dot{y}_{t}=\frac{1}{\tau}\left[u\left(x_{t}, y_{t}\right)-\delta \frac{\partial u}{\partial x}\left(x_{t}, y_{t}\right) \dot{x}_{t}-\delta \frac{\partial u}{\partial y}\left(x_{t}, y_{t}\right) \dot{y}_{t}\right] \sin \phi_{t} \\ \phi_{t}=\sqrt{\frac{2}{\tau}} W_{t} .\end{array}\right.$

Importantly, in this form, they make sense also for negative $\delta$. The stationary density (if it exists) of finding the robot at a position $(x, y)$ is

$$
\rho_{0}(x, y)=\frac{1}{N u(x, y)^{1+\frac{\delta}{\tau}}} .
$$

(a)
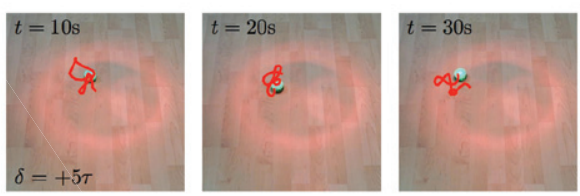

(b)
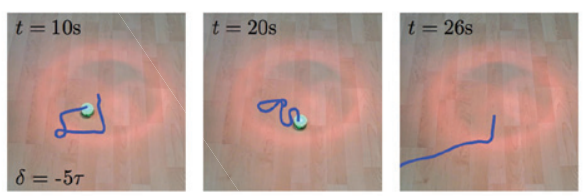

(c)

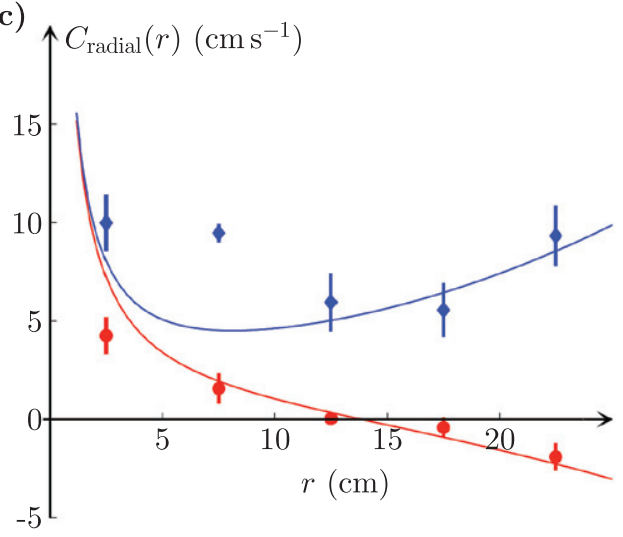

Figure 12. Effect of sensorial delay on the behavior of an autonomous agent. The long-term behavior of a robot in the light intensity gradient generated by an infrared lamp changes depending on the delay with which it adjusts its speed in response to the sensorial input, i.e. the measured total light intensity. (a) For positive delays $(\delta=+5 \tau)$, the tendency of the robot to move towards the high-intensity (low-speed) regions is enhanced,

when compared to the case without delay. (b) For negative delays $(\delta=-5 \tau)$ the robot tends to move towards the low-intensity (highspeed) regions. In both cases, the trajectories are shown for a period of $5 \mathrm{~s}$ preceding the time indicated on the plot and the robot is at the final position. (c) Radial drift calculated from a 40 min trajectory for the cases of positive (circles) and negative (diamonds) delays The solid lines correspond to the theoretically predicted radial drifts. Adapted with permission from [62].

which represents a probability density if it is an integrable function (and in this case we choose the normalizing constant $N$ so that the integral of $\rho_{0}$ equals 1$)$. An outline of the mathematical derivation of equation (33) is provided in appendix $E$. The critical role of the condition $\delta=-\tau$ is clearly seen from this formula: as long as $\delta>-\tau$, the density is larger where $u$ is smaller, but the relation reverses for $\delta<-\tau$. Therefore, for $\delta>-\tau$ the robot tends to spend more time in the region where $u$ is smaller, in agreement with the natural intuition: once it gets there, it slows down, so it takes it longer to get out. However, the two time scales interact in a complicated way and the above intuition correctly predicts what is happening only when the delay is positive or when it is negative, but its magnitude is not too big compared to the noise correlation time. Beyond this value (i.e. for $\delta<-\tau$ ) the behavior changes qualitatively. This effect is seen clearly in the experiment as well as in numerical calculations [62], as shown in figure 12.

The second part of the experiment studies many robots that influence each other's motion through light fields each 


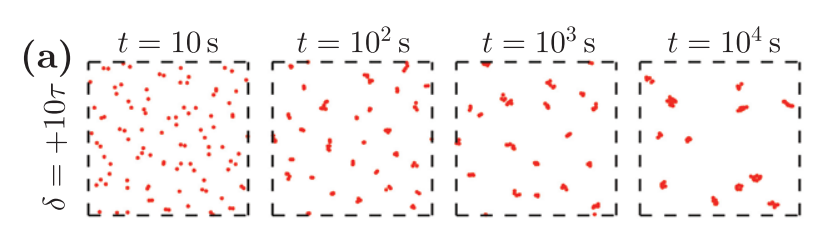

(b)

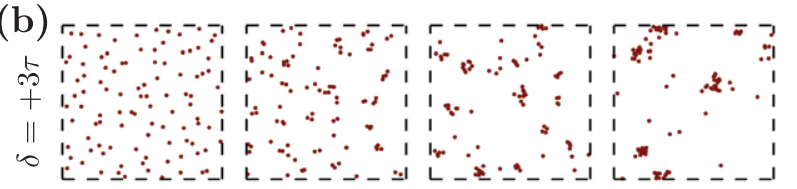

(c)

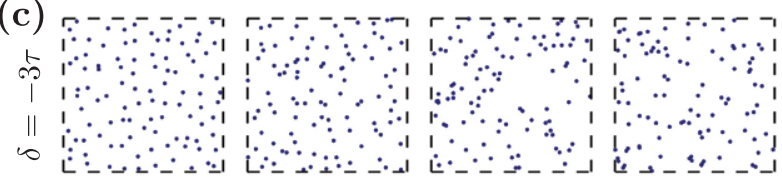

(d)

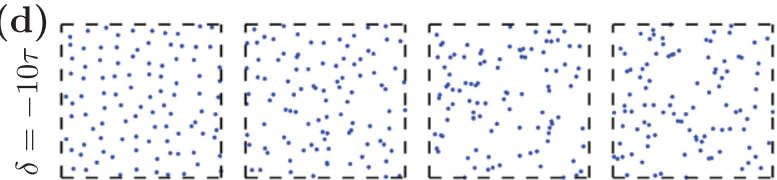

Figure 13. Clustering and segregation in a swarm of autonomous agents as a function of sensorial delay. Simulation of the long-term behavior of an ensemble of 100 autonomous agents that emit a radially decaying intensity field and adjust their speed depending on the measured local intensity. Depending on the sensorial delay, the long-term behavior and large-scale organization are signifiicantly different. (a), (b) In the case of positive delays, the agents come together and form metastable clusters. (c), (d) In the case of negative delays, they explore the space, staying away from each other. Adapted with permission from [62].

of them creates, as shown in figure 13. Each robot's speed is a function of the total light intensity at its location (with the time delay as above). It is observed that when $\delta>-\tau$, the robots tend to aggregate, but for $\delta<-\tau$ they stay away from each other. This collective effect is a reflection of the single robot's behavior studied above: for delays greater than $-\tau$ a robot spends more time in the vicinity of other robots, which decreases its velocity by creating a stronger cumulative light field, and for delays smaller than $-\tau$ a robot spends more time away from other robots, where its velocity is larger.

\section{Applications, future work and perspectives}

As we have seen in the previous sections, there is often a need to derive effective and tractable mathematical models that reduce the number of degrees of freedom of real systems while still representing their complex nature. In fact, the exact modeling of phenomena discussed in this review would require access to their microscopic dynamics, whose time scales are typically much shorter than the observable time scales. A further reduction can be obtained by considering limits in which one or more natural time scales of the problem go to zero. We have also seen that the presence of multiplicative noise (in its multifaceted forms) leads to the appearance of noise-induced drifts in the effective SDEs. Importantly, recent experiments have been able to measure these noise-induced drifts and their consequences in the case of Brownian particles in thermal equilibrium with a heat bath $[12,26,40,41]$ and in the case of electric circuits [22, 27]. Even more importantly, at least one subsequent experiment [62] puts forward a concrete application, by using a noise-induced drift to control the long-term behavior of autonomous agents.

We expect future research to focus on noise-induced drifts and on their dramatic consequences in many cases where SDEs with multiplicative noise are routinely employed to predict the behavior and evolution of complex physical, chemical, biological and economic phenomena. In particular, there is a need to study in more detail the nature and significance of noise-induced drifts in multidimensional systems. In fact, while several theoretical works have dealt with the multidimensional case, all experiments performed until now focus on noise-induced drifts emerging in effectively one-dimensional systems, i.e. systems where the number of effective degrees of freedom has been reduced to one, even when they are intrinsically multidimensional. For example, in [62] the motion of the robots occurs in a plane, but the effective noise-induced drift is measured only along the radial coordinate.

Here we provide a list of topics of interest, focusing on effects that are important for applications and/or that can be verified experimentally.

- More realistic experimental model systems. Electrical circuits are relatively easily controllable physical systems with damping and noise. As such, they provide a natural class of systems whose parameters can be manipulated to test the theory, e.g. to observe noise-induced bifurcations [22] and transitions [27]. However, they are also relatively simple physical systems. It will therefore be crucial to move towards experimentation in more relevant and realistic systems. For example, biological systems can be investigated starting from simple bacterial colonies reacting to a time-varying environment in order to study whether, e.g. noise-induced bifurcation in the population dynamics may occur, and moving at a later stage towards more complex ecosystems. Economic systems can be analyzed by using available econometric data; for example, it would be fascinating to study the possibility that booms and bursts in the stock market might be due to a noise-induced transition similar to the one described in section 3.3.

- Effect of multiplicative noise on steady-state distributions. As we have seen in section 2 and, in particular, in figure 4 , the presence of a noise-induced drift changes the stationary distribution of an SDE system (if it has one). While for systems satisfying the fluctuation-dissipation relation, e.g. in thermal equilibrium, potential landscape and steady-state (or, in this case, equilibrium) distribution are connected by the Boltzmann statistics [25], this is not necessarily the case for other systems [46]. Things become particularly tantalizing when considering multidimensional systems, where also non-conservative (e.g. magnetic) forces may be present. Overall, it will be interesting to explore the interplay between multiplicative noise, noise-induced drifts, non-conservative forces, and steady-state probability distributions both from theoretical and experimental perspectives. 
- Noise-induced bifurcations. Noise-induced drifts can radically modify the properties of a dynamical system, making it undergo a bifurcation. Interestingly, one of the first works studying experimentally systems with multiplicative noise [22] addressed precisely the issue of how the transition from a monostable to a bistable behavior in a noisy electric circuit was affected by the presence of multiplicative noise (section 3.3 and figure 9). Also, a system similar to a van der Pol oscillator has been recently theoretically studied in this context [65]. These studies constitute a good starting point to explore more complex situations. In particular, we are planning to identify conditions under which the presence of noise and state-dependent damping induces specific types of bifurcations, e.g. saddle-node or Hopf bifurcations. Furthermore, we are planning to study models of population dynamics, including, e.g. effects of randomness on Lotka-Volterra-type equations (see, e.g. Tang et al [66]). We emphasize that the very interesting theory of stochastic bifurcations (see, e.g. [67, chapter 9]) is concerned with bifurcations of vector fields (or their absence) under adding individual realizations of the noise and thus has a different focus from the one proposed here. Similarly, Arnold [68] studies stability of equilibria of dynamical systems perturbed by individual noise realizations, rather than modified by the noise-induced drifts considered here.

- Noise-induced drifts in thermophoresis. While it has already been suggested that noise-induced drifts might play a role in thermophoresis [30, 49], this is a subject that still needs to be investigated in detail both theoretically and experimentally. In particular, in the presence of a colored noise, the damping term should be an integral of contributions with different delay times and one should study the noise-induced drift in the resulting stochastic integro-differrential equations.

- Noise-induced drift in stochastic thermodynamics. Despite some very recent theoretical works [69-71], the interplay between noise-induced drifts and stochastic thermodynamics is still a largely unexplored and tantalizing field of research.

- Noise-induced drifts in noise-induced phenomena. Noise plays a crucial (and constructive) role in many phenomena such as Kramers transitions [51], stochastic resonance [72], and Brownian ratchets [73]. It will be fascinating to explore how multiplicative noises and noise-induced drifts can affect such phenomena.

- Entropy production in the small-mass limit. Entropy production in stochastic systems has been a subject of numerous recent works (a systematic exposition is given in [74]). Celani et al [75] discuss the behavior of entropy production for the equation equivalent to SDE (23) with constant damping (and zero external force), where there is no noise-induced drift. We propose to study entropy production in the general SDEs (25). This may lead to a variational characterization of the noise-induced drift.
- Noise-induced drifts in curved spaces. Another direction of future work is concerned with the diffusion of Brownian and active Brownian particles $[63,64]$ on surfaces. In addition to its intrinsic mathematical interest and beauty, diffusion on surfaces occurs naturally in biology (e.g. molecular complexes on a cell membrane, white blood cells on the surface of an alveolus) and in physics (e.g. colloids trapped on a membrane or interface). For example, Polettini [76, 77] has recently suggested an analogy between the motion of a Brownian particle constrained in a curved geometry (more specifically in one-dimensional curves embedded in a space of an arbitrary dimension) and in a temperature gradient. The techniques outlined in this review allow one to study the zero-mass and related limits of equations describing such systems. In particular, we are planning two theoretical projects. In the first one, we will present the Wiener process (Brownian motion) on a manifold as a zero-mass limit of an inertial system, justifying its use in mathematical modeling of overdamped systems of surface diffusion. In the second project, we will consider a particle moving on a two-dimensional surface by inertia, with rapid random changes of direction (as in the example discussed in section 3.4). Considering an active particle that rotates around its center, we aim to show that in the limit of fast rotations, the particle's dynamics is described by the Wiener process in agreement with the general result of [78]. To complete these two projects we will couple the techniques presented here with those of stochastic differential geometry $[79,80]$. Finally, we are planning a numerical study of diffusion on two-dimensional surfaces in the presence of interesting geometry, resulting in longterm particle trapping, similarly to the results reported (in a different context) by Chepizhko and Peruani [81].

- Quantum noise-induced drift. Another interesting research direction is to study noise-induced drifts in open quantum systems. The dynamics of such systems in the Markov approximation is described by quantum Langevin equations (in the Heisenberg picture). We are planning to conduct an analysis of these equations, similar to the integration-by-parts technique described here. In some systems, the master equation (analog of the Kolmogorov equation of classical theory) may be more amenable to analysis, patterned in this case on the classical multiscale analysis (homogenization). Among others, we will study quantum Brownian particles whose coupling to the environment depends on position. A physical realization of such system is the motion of an impurity atom interacting with a Bose-Einstein condensate. See also the recent review by Massignan et al [37] and the references therein.

In conclusion, the study of multiplicative noise and of the associated noise-induced drifts has recently become an active and fertile field of research. It opens several interesting avenues towards studying new phenomena and offers exciting future research directions, with implications for both fundamental science and potential technological applications. 


\section{Acknowledgments}

We thank our collaborators on various parts of the projects presented here: $\mathrm{C}$ Bechinger, $\mathrm{T}$ Brettschneider, O Duman, L Helden, D Herzog, S Hottovy, A McDaniel, M Mijalkov and $G$ Pesce. We are also grateful to several colleagues for discussions and important comments: P Ao, V Blickle, T Franosch, A Gambassi, K Gawędzki, T Kurtz, M Lewenstein, R Mannella, J Mehl, M Rauscher, P Reimann, U Seifert, S R S Varadhan and J Watkins. GV has been partially financed by Marie Curie Career Integration Grant (MC-CIG) PCIG11 GA-2012-321726 and a Distinguished Young Scientist award of the Turkish Academy of Sciences (TÜBA). JW has been partially funded by NSF grant DMS 131271 and a part of his work on this article was supported by NSF under grant DMS 1440140 while he was in residence at the Mathematical Sciences Research Institute in Berkeley during the Fall 2015 semester.

\section{Appendix A. Finite-difference (FD) numerical simulations}

The numerical integration of SDEs is discussed in detail in [4]; here we provide a primer on how to integrate SDEs with multiplicative noise accounting for the integration convention.

In the FD integration of an ordinary differential equation (ODE), the continuous-time solution $x(t)$ of the ODE is approximated by a discrete-time sequence $x_{n}$, which is the solution of the corresponding FDE evaluated at regular time steps $t_{n}=n \Delta t$. If $\Delta t$ is sufficiently short, $x_{n} \approx x\left(t_{n}\right)$. For example, in the case of a $1^{\text {st }}$ order ODE, the FDE is obtained by perfoming the following substitutions:

$$
\begin{aligned}
x(t) & \mapsto x_{n}, \\
\dot{x}(t) & \mapsto \frac{x_{n+1}-x_{n}}{\Delta t} .
\end{aligned}
$$

The solution is then obtained by solving the resulting FDE recursively for $x_{n+1}$, using the previous value $x_{n}$ as the initial condition.

Let us now consider the SDE

$$
\mathrm{d} x_{t}=g\left(x_{t}\right) \mathrm{d} t+\sigma\left(x_{t}\right) \circ_{\alpha} \mathrm{d} W_{t},
$$

where the noise term is to be integrated with the convention $\alpha$. As we have seen at the end of section 2, the SDE (A.1) is equivalent to

$$
\mathrm{d} x_{t}=g\left(x_{t}\right) \mathrm{d} t+\alpha \sigma\left(x_{t}\right) \frac{\mathrm{d} \sigma\left(x_{t}\right)}{\mathrm{d} x} \mathrm{~d} t+\sigma\left(x_{t}\right) \mathrm{d} W_{t},
$$

where the multiplicative noise term is an Itô integral. The numerical integration of the first two terms on the right-hand side of SDE (A.2) is straightforward and can be performed as for the case of ODEs. In the FDE, the noise term, i.e. $\sigma\left(x_{t}\right) \mathrm{d} W_{t}$, is replaced by $\sigma\left(x_{n}\right) w_{n}$, where $w_{n}$ is a Gaussian random number with zero mean and variance $1 / \Delta t$. Thus, the resulting FDE corresponding to $\operatorname{SDE}$ (A.1) (and SDE (A.2)) is

$$
x_{n+1}=x_{n}+g\left(x_{n}\right) \Delta t+\alpha \sigma\left(x_{n}\right) \frac{\mathrm{d} \sigma\left(x_{n}\right)}{\mathrm{d} x} \mathrm{~d} t+\sigma\left(x_{n}\right) w_{n} .
$$

This approach can be straightforwardly generalized to vectorial systems.

\section{Appendix B. Derivation of the spurious drift of a Brownian particle}

SDE (14) is equivalent to the system

$\left\{\begin{array}{l}\mathrm{d} x_{t}=v_{t} \mathrm{~d} t, \\ \mathrm{~d} v_{t}=\frac{F\left(x_{t}\right)}{m} \mathrm{~d} t-\frac{k_{\mathrm{B}} T}{m D\left(x_{t}\right)} v_{t} \mathrm{~d} t+\frac{k_{\mathrm{B}} T \sqrt{2}}{m \sqrt{D\left(x_{t}\right)}} \mathrm{d} W_{t},\end{array}\right.$

where $W_{t}=\int_{0}^{t} \eta_{s} \mathrm{~d} s$ is the time integral of the white noise ${ }^{7}$. The evolution of a probability density $\rho(x, v)$ under the stochastic dynamics defined by this system is described by the FokkerPlanck (in mathematics literature: forward Kolmogorov) equation

$\rho_{t}=\frac{\left(k_{\mathrm{B}} T\right)^{2}}{m^{2} D\left(x_{t}\right)} \rho_{v v}-v \rho_{x}-\frac{F(x)}{m} \rho_{v}+\frac{k_{\mathrm{B}} T}{m D(x)}(\rho v)_{v}$,

where the subscripts denote partial derivatives. To find the steady-state probability density, we need to solve this equation with the left-hand side equal to zero. A direct calculation shows that it is satisfied by the Boltzmann-Gibbs probability distribution given by equation (16).

We now outline the derivation of the correct limiting SDE for $m \rightarrow 0$ and the corresponding noise-induced drift. We start by rewriting the system (B.1) as

$$
\begin{aligned}
\mathrm{d} x_{t} & =v_{t} \mathrm{~d} t \\
& =\frac{F\left(x_{t}\right) D\left(x_{t}\right)}{k_{\mathrm{B}} T} \mathrm{~d} t+\sqrt{2 D\left(x_{t}\right)} \mathrm{d} W_{t}-\frac{m}{k_{\mathrm{B}} T} D\left(x_{t}\right) \mathrm{d} v_{t}
\end{aligned}
$$

In integral form, SDE (B.3) becomes

$$
\begin{aligned}
x_{t}=x_{0} & +\int_{0}^{t} \frac{F\left(x_{s}\right) D\left(x_{s}\right)}{k_{\mathrm{B}} T} \mathrm{~d} s \\
& +\int_{0}^{t} \sqrt{2 D\left(x_{s}\right)} \mathrm{d} W_{s} \\
& -\int_{0}^{t} \frac{m}{k_{\mathrm{B}} T} D\left(x_{s}\right) \mathrm{d} v_{s},
\end{aligned}
$$

where the first integral term on the right-hand side is the contribution due to the deterministic (external) forces and the next term is an Itô integral. In order to derive the noiseinduced drift, we will study the limiting behavior for $m \rightarrow 0$ of the last term. We start by integrating it by parts, obtaining

$$
\begin{aligned}
-\int_{0}^{t} \frac{m}{k_{\mathrm{B}} T} D\left(x_{s}\right) \mathrm{d} v_{s}= & \frac{1}{k_{\mathrm{B}} T}\left[m v_{0} D\left(x_{0}\right)-m v_{t} D\left(x_{t}\right)\right] \\
& +\int_{0}^{t} \frac{m}{k_{\mathrm{B}} T} \frac{\mathrm{d} D\left(x_{s}\right)}{\mathrm{d} x} v_{s}^{2} \mathrm{~d} s .
\end{aligned}
$$

Using equation (17), the boundary terms in equation (B.5) go to zero with $m \rightarrow 0$ and, replacing the kinetic energy $\left(\frac{1}{2} m v_{s}^{2}\right)$ by

\footnotetext{
${ }^{7}$ Note that in SDEs (B.1) there is no multiplicative noise because the noise term for $v_{t}$ is multiplied by a function of $x_{t}$ (not of $v_{t}$ ).
} 
its average $\left(\frac{1}{2} k_{\mathrm{B}} T\right)$ in the last integral, we obtain the effective SDE (18).

We emphasize that the averaging of the kinetic energy is far from trivial and needs a careful justification; a sketch of the argument (in a more general case) will be given in section 3.2. The physical picture is that the velocity $v_{t}$ is a fast variable that homogenizes in the $m \rightarrow 0$ limit. The term adiabatic elimination is also used in literature to describe this phenomenon. The above result was proven rigorously by Hottovy et al [48] and by Herzog et al [82]. Related results were obtained earlier by Hänggi [19] and by Sancho et al [39]. Interestingly, Marchesoni [83] has recently suggested that the spurious drift at zero current could possibly provide an option to design a Maxwell demon.

\section{Appendix C. Derivation of noise-induced drift for systems not satisfying the fluctuation-dissipation relation}

To analyze the general SDE (23), we follow the argument in [48], concentrating on the main steps and leaving out technical details and estimates. The first step in the derivation of the limiting equation is the same as in the special case studied in appendix B: introducing the velocity $v_{t}$, we rewrite SDE (24) as

$\mathrm{d} x_{t}=v_{t} \mathrm{~d} t=\frac{F\left(x_{t}\right)}{\gamma\left(x_{t}\right)} \mathrm{d} t+\frac{\sigma\left(x_{t}\right)}{\gamma\left(x_{t}\right)} \mathrm{d} W_{t}-\frac{m}{\gamma\left(x_{t}\right)} \mathrm{d} v_{t}$.

The first two terms on the right-hand side do not depend explicitly on $m$ and thus remain unchanged in the limit $m \rightarrow 0$. To derive the limiting contribution of the third term, we use the product rule:

$$
\frac{m}{\gamma\left(x_{t}\right)} \mathrm{d} v_{t}=m d\left(v_{t} \gamma\left(x_{t}\right)\right)-d\left(\frac{m}{\gamma\left(x_{t}\right)}\right) v_{t} .
$$

While the equipartition theorem no longer holds in this generality, we will show that the fast oscillations of the velocity allow to replace in the integrals the expression $m v_{t}^{2}$ by a function of $x_{t}$ (homogenization or adiabatic elimination of the fast variable $v_{t}$ [54]). This is done by first showing that $m v_{t}$ converges to zero as $m \rightarrow 0$ (a technical step, involving careful estimates [48]). It follows that in the integral form of the last equation, i.e.

$\int_{0}^{t} \frac{m}{\gamma\left(x_{s}\right)} \mathrm{d} v_{s}=m\left[\frac{v_{t}}{\gamma\left(x_{t}\right)}-\frac{v_{0}}{\gamma\left(x_{0}\right)}\right]-\int_{0}^{t} d\left(\frac{m}{\gamma\left(x_{s}\right)}\right) v_{s}$,

the first term on the right-hand side vanishes in the limit. The integrand in the second term equals $\gamma\left(x_{t}\right)^{-2} \frac{\mathrm{d} \gamma\left(x_{t}\right)}{\mathrm{d} x} m v_{t}^{2}$. To find its homogenization limit, we study the expression $d\left[\left(m v_{t}\right)^{2}\right]$. On the one hand, this quantity becomes zero when $m \rightarrow 0$. On the other hand, using the Itô product formula [5], we have

$$
d\left(m v_{t} \cdot m v_{t}\right)=2 m v_{t} d\left(m v_{t}\right)+\left[d\left(m v_{t}\right)\right]^{2} .
$$

Substituting for $m \mathrm{~d} v_{t}$ the expression on the right-hand side of SDE (23), we obtain $d\left[\left(m v_{t}\right)^{2}\right]=2 m v_{t}\left[F\left(x_{t}\right) \mathrm{d} t-\gamma\left(x_{t}\right) v_{t} \mathrm{~d} t+\sigma\left(x_{t}\right) \mathrm{d} W_{t}\right]+\sigma\left(x_{t}\right)^{2} \mathrm{~d} t$.

The first and third terms on the right-hand side converge to zero, since $m v_{t}$ does (the rigorous argument again requires some care [48]). Since in the limit $m \rightarrow 0$, the whole expression converges to zero, it follows that $m v_{t}^{2} \mathrm{~d} t$ is asymptotically equivalent to $\frac{\sigma\left(x_{t}\right)^{2}}{2 \gamma\left(x_{t}\right)} \mathrm{d} t$. We thus obtain from equation (C.3)

$$
\int_{0}^{t} \frac{m}{\gamma\left(x_{s}\right)} \mathrm{d} v_{s} \rightarrow \int_{0}^{t} \frac{\sigma\left(x_{t}\right)^{2}}{2 \gamma\left(x_{t}\right)^{3}} \frac{\mathrm{d} \gamma\left(x_{t}\right)}{\mathrm{d} x} \mathrm{~d} t
$$

in the zero mass limit. Substituted into SDE (C.1), this gives the limiting SDE (24).

The method described above can be adapted to the multidimensional case, i.e. to derive the limit of SDE (25) as the mass goes to zero. The main idea is the same as in the onedimensional case, but calculations become more complicated and the description of the noise-induced drift is more involved: it is expressed using a unique solution of a matrix equation (the Lyapunov equation). The final result becomes more implicit, since the solution of the Lyapunov equation is, in general, expressed as an integral over an auxiliary parameter. In an important class of cases, the Lyapunov equation has an explicit solution and the limiting equation becomes explicit as well.

The precise form of the limiting equation is

$\mathrm{d} \boldsymbol{x}_{t}=\left[\boldsymbol{\gamma}^{-1}\left(\boldsymbol{x}_{t}\right) \boldsymbol{F}\left(\boldsymbol{x}_{t}\right)+\boldsymbol{S}\left(\boldsymbol{x}_{t}\right)\right] \mathrm{d} t+\gamma^{-1}\left(\boldsymbol{x}_{t}\right) \boldsymbol{\sigma}\left(\boldsymbol{x}_{t}\right) \mathrm{d} \boldsymbol{W}_{t}$,

where $\boldsymbol{S}\left(\boldsymbol{x}_{t}\right)$ is the noise-induced drift whose $i^{\text {th }}$ component equals

$$
S_{i}(\boldsymbol{x})=\sum_{j, l} \frac{\partial}{\partial x_{l}}\left[\left(\gamma^{-1}\right)_{i j}(\boldsymbol{x})\right] J_{j l}(\boldsymbol{x}),
$$

and $\boldsymbol{J}$ is the matrix solving the Lyapunov equation

$$
\boldsymbol{J} \boldsymbol{\gamma}^{*}+\gamma \boldsymbol{J}=\boldsymbol{\sigma} \boldsymbol{\sigma}^{*}
$$

When all eigenvalues of $\gamma$ have positive real parts, the unique solution is given by the formula [84, chapter 11]

$$
\mathbf{J}=\int_{0}^{\infty} \mathrm{e}^{-y \gamma} \boldsymbol{\sigma} \boldsymbol{\sigma}^{*} \mathrm{e}^{-y \gamma^{*}} \mathrm{~d} y .
$$

Note that when $\gamma=\gamma^{*}$ commutes with $\sigma \sigma^{*}$, the solution of the Lyapunov equation is explicitly given by $\boldsymbol{J}=\frac{1}{2} \gamma^{-1} \boldsymbol{\sigma} \boldsymbol{\sigma}^{*}$.

\section{Appendix D. Derivation of the noise-induced drift for systems with colored noise and delayed feedback}

To derive the limiting SDE (29), we approximate the delay equation by an SDE without delay and apply the method based on integration by parts outlined in appendix C. First, we define a time-shifted process

$$
z_{t}=x_{t-\delta}
$$

and rewrite the equation as

$$
\mathrm{d} z_{t+\delta}=-k z_{t+\delta}+\sigma F\left(z_{t}\right) \eta_{t}^{\tau} \mathrm{d} t
$$

Introducing the process 


$$
v_{t}=\frac{\mathrm{d} z_{t}}{\mathrm{~d} t}
$$

we use approximations

$$
z_{t+\delta} \approx z_{t}+\delta v_{t}
$$

and, accordingly,

$$
\mathrm{d} z_{t+\delta} \approx \mathrm{d} z_{t}+\delta \mathrm{d} v_{t}
$$

Substituting these expressions into SDE (28) and solving for $\mathrm{d} v_{t}$, we obtain the system

$\left\{\begin{array}{l}\mathrm{d} z_{t}=v_{t} \mathrm{~d} t \\ \mathrm{~d} v_{t}=-\frac{1}{\delta} k z_{t} \mathrm{~d} t-\left(\frac{1}{\delta}+k\right) v_{t} \mathrm{~d} t+\frac{1}{\delta} \eta_{t}^{\tau} \mathrm{d} t .\end{array}\right.$

This system can be studied using the method outlined in appendix $\mathrm{C}$ : we add the equations describing the process $\eta^{\tau}$ to the system and apply the general method of [48] to identify the limiting system which matches the experimental results.

In [61], the same method is applied to a much more general system of delayed SDE driven by several colored noises with couplings that are functions of the delayed dynamical variables:

$$
\mathrm{d} \boldsymbol{x}_{t}=\boldsymbol{f}\left(\boldsymbol{x}_{t}\right) \mathrm{d} t+\boldsymbol{g}\left(\boldsymbol{x}_{t-\delta}\right) \boldsymbol{\eta}_{t}^{\tau} \mathrm{d} t,
$$

where $\boldsymbol{x}_{t}=\left(x_{t}^{1}, \ldots, x_{t}^{i}, \ldots, x_{t}^{m}\right)^{\mathrm{T}}$ is the state vector (the superscript ' $\mathrm{T}$ ' denotes transposition), $\boldsymbol{f}\left(\boldsymbol{x}_{t}\right)=\left(f^{1}\left(\boldsymbol{x}_{t}\right), \ldots, f^{i}\left(\boldsymbol{x}_{t}\right), \ldots, f^{m}\left(\boldsymbol{x}_{t}\right)\right)^{\mathrm{T}}$ is a vector-valued function describing the deterministic part of the dynamical system,

$\boldsymbol{g}\left(\boldsymbol{x}_{t-\delta}\right)=\left[\begin{array}{ccccc}g^{11}\left(\boldsymbol{x}_{t-\delta}\right) & \ldots & g^{1 j}\left(\boldsymbol{x}_{t-\delta}\right) & \ldots & g^{1 n}\left(\boldsymbol{x}_{t-\delta}\right) \\ \vdots & \ddots & \vdots & \ddots & \vdots \\ g^{i 1}\left(\boldsymbol{x}_{t-\delta}\right) & \ldots & g^{i j}\left(\boldsymbol{x}_{t-\delta}\right) & \ldots & g^{i n}\left(\boldsymbol{x}_{t-\delta}\right) \\ \vdots & \ddots & \vdots & \ddots & \vdots \\ g^{m 1}\left(\boldsymbol{x}_{t-\delta}\right) & \ldots & g^{m j}\left(\boldsymbol{x}_{t-\delta}\right) & \ldots & g^{m n}\left(\boldsymbol{x}_{t-\delta}\right)\end{array}\right]$

is a matrix-valued function, $\boldsymbol{x}_{t-\delta}=\left(x_{t-\delta_{1}}^{1}, \ldots, x_{t-\delta_{i}}^{i}, \ldots, x_{t-\delta_{m}}^{m}\right)^{\mathrm{T}}$ is the delayed state vector (note that each component is delayed by a possibly different amount $\delta_{i}>0$ ), and $\boldsymbol{\eta}_{t}^{\tau}=\left(\eta_{t}^{\tau, 1}, \ldots, \eta_{t}^{\tau, j}, \ldots, \eta_{t}^{\tau, n}\right)^{\mathrm{T}}$ is a vector of independent noises $\eta^{\tau, j}$, where $\eta^{\tau, j}$ are colored noises (harmonic noises [85]) with characteristic correlation times $\tau_{j}$, as described in detail in [27]. We study the limit of this SDE as the parameters $\delta_{i}$ and $\tau_{j}$ all go to zero at the same rate, i.e. $\delta_{i}=c_{i} \epsilon, \tau_{j}=k_{j} \epsilon$ where $c_{i}$ and $k_{j}$ are constants and $\epsilon \rightarrow 0$. Using a modification of the method outlined in appendix $\mathrm{C}$, we obtain the limiting system

$$
\begin{aligned}
\mathrm{d} y_{t}^{i}= & f^{i}\left(\boldsymbol{y}_{t}\right) \mathrm{d} t+\sum_{j} g^{i j}\left(\boldsymbol{y}_{t}\right) \mathrm{d} W_{t}^{j} \\
& +\underbrace{\sum_{p, j} g^{p j}\left(\boldsymbol{y}_{t}\right) \frac{\partial g^{i j}\left(\boldsymbol{y}_{t}\right)}{\partial y_{p}} \frac{1}{2}\left(1+\frac{c_{p}}{k_{j}}\right)^{-1} \mathrm{~d} t .}_{\text {nose-induced drifts }}
\end{aligned}
$$

The noise-induced drift terms are again of the Itô-Stratonovich correction type, entering with coefficients that are explicit functions of the $c_{i}$ and $k_{j}$. See also [90] for a more precise result replacing $\left(1+\frac{c_{p}}{k_{j}}\right)$ by $\exp \left(-\frac{c_{p}}{k_{j}}\right)$
Appendix E. Derivation of noise-induced drift for systems with sensorial delay

In order to derive equation (33), we will study the approximate SDEs (32) and derive limiting SDEs for $x_{t}$ and $y_{t}$ in the limit when $\tau$ and $\delta$ go to zero at the same rate. It will be seen that, within the approximation discussed below, these equations reproduce the experimental results obtained in [62] and discussed in section 3.4, including a qualitative change of the robot's behavior at $\delta=-\tau$.

Solving the first two SDEs (32) for $\dot{x}_{t}$ and $\dot{y}_{t}$, we obtain the system

$$
\left\{\begin{array}{l}
\dot{x}_{t}=\frac{\frac{1}{\sqrt{\tau}} u\left(x_{t}, y_{t}\right) \cos \phi_{t}}{1+\frac{\delta}{\sqrt{\tau}}\left(\frac{\partial u}{\partial x}\left(x_{t}, y_{t}\right) \cos \phi_{t}+\frac{\partial u}{\partial y}\left(x_{t}, y_{t}\right) \sin \phi_{t}\right)}, \\
\dot{y}_{t}=\frac{\frac{1}{\sqrt{\tau}} u\left(x_{t}, y_{t}\right) \sin \phi_{t}}{1+\frac{\delta}{\sqrt{\tau}}\left(\frac{\partial u}{\partial x}\left(x_{t}, y_{t}\right) \cos \phi_{t}+\frac{\partial u}{\partial y}\left(x_{t}, y_{t}\right) \sin \phi_{t}\right)}, \\
\phi_{t}=\sqrt{\frac{2}{\tau}} W_{t},
\end{array}\right.
$$

which, assuming that $\delta / \sqrt{\tau} \ll 1$, we approximate further by

$$
\left\{\begin{aligned}
\dot{x}_{t}= & \frac{1}{\sqrt{\tau}} u\left(x_{t}, y_{t}\right) \cos \phi_{t} \\
& \times\left[1-\frac{\delta}{\sqrt{\tau}}\left(\frac{\partial u}{\partial x}\left(x_{t}, y_{t}\right) \cos \phi_{t}+\frac{\partial u}{\partial y}\left(x_{t}, y_{t}\right) \sin \phi_{t}\right)\right] \\
\dot{y}_{t}= & \frac{1}{\sqrt{\tau}} u\left(x_{t}, y_{t}\right) \sin \phi_{t} \\
& \times\left[1-\frac{\delta}{\sqrt{\tau}}\left(\frac{\partial u}{\partial x}\left(x_{t}, y_{t}\right) \cos \phi_{t}+\frac{\partial u}{\partial y}\left(x_{t}, y_{t}\right) \sin \phi_{t}\right)\right] \\
\phi_{t}= & \sqrt{\frac{2}{\tau}} W_{t} .
\end{aligned}\right.
$$

As mentioned earlier, the above system is meaningful for both positive and negative $\delta$; in fact, it is for "negative time delay' $(\delta<0)$ that we see the most interesting phenomena. We are going to study its limit when $\delta$ and $\tau$ go to zero at the same rate, which is consistent with our earlier assumption $\delta / \sqrt{\tau} \ll 1$.

The methods described in the previous appendices do not apply to the above system. Instead, we use the multiscale method, whose mathematical details and foundations can be found in [54] or in [53]. The essence of the method is to study a partial differential equation (the backward Kolmogorov equation) associated with the system (E.2), take its limit as $\epsilon \rightarrow 0$, and recover the limiting SDE system from the result. The calculations are involved and we refer the reader to [62] for details. The limiting Kolmogorov equation is

$\frac{\partial \rho_{0}}{\partial t}=\frac{1}{2}\left(1-\frac{\delta}{\tau}\right) u\left(\frac{\partial u}{\partial x} \frac{\partial \rho_{0}}{\partial x}+\frac{\partial u}{\partial y} \frac{\partial \rho_{0}}{\partial y}\right)+\frac{u^{2}}{2} \Delta \rho_{0}$.

To obtain a meaningful limit, we scaled the velocity by $\sqrt{\tau}$. This means that to compare the result with experiment, we 
have to substitute $u=\sqrt{\tau} v$ and rewrite the equation in terms of $v$ :

$\frac{\partial \rho_{0}}{\partial t}=\frac{\tau}{2}\left(1-\frac{\delta}{\tau} v\right)\left(\frac{\partial v}{\partial x} \frac{\partial \rho_{0}}{\partial x}+\frac{\partial v}{\partial y} \frac{\partial \rho_{0}}{\partial y}\right)+\tau \frac{v^{2}}{2} \Delta \rho_{0}$.

According to diffusion theory [5], the limiting SDEs for $x_{t}$ and $y_{t}$, which correspond to this Kolmogorov equation, are

$$
\left\{\begin{array}{r}
\mathrm{d} x_{t}=\frac{\tau}{2}\left(1-\frac{\delta}{\tau}\right) v\left(x_{t}, y_{t}\right) \frac{\partial v}{\partial x}\left(x_{t}, y_{t}\right) \mathrm{d} t \\
+\sqrt{\tau} v\left(x_{t}, y_{t}\right) \mathrm{d} W_{t}^{(1)}, \\
\mathrm{d} y_{t}=\frac{\tau}{2}\left(1-\frac{\delta}{\tau}\right) v\left(x_{t}, y_{t}\right) \frac{\partial v}{\partial y}\left(x_{t}, y_{t}\right) \mathrm{d} t \\
+\sqrt{\tau} v\left(x_{t}, y_{t}\right) \mathrm{d} W_{t}^{(2)},
\end{array}\right.
$$

where $W^{(1)}$ and $W^{(2)}$ are independent Wiener processes. Two remarks are in order. First, the angular variable $\phi$ is no longer present in the limiting equations: similarly to $v_{t}$ in the Brownian motion case, it is a fast variable that gets adiabatically eliminated in the limit. Second, the limiting SDEs involve two independent sources of noise, while only one was present in the original ones. This system correctly reproduces the limiting distribution (statistics) of the paths solving the original system whose asymptotics is, however, not studied here for individual realizations of the noise. Rather, for each value of $\tau$ and $\delta$ (with the ratio $\delta / \tau$ kept constant) we consider the distribution of the solutions and study the limit of this distribution, i.e. of the probability measure on the path space. This mode of convergence is called weak [86]. The presence of two independent noise sources in the limiting system makes it unlikely that one can prove a stronger convergence statement, including any information about the solutions' behavior for a fixed realization of the noise. In particular, such a statement would require a natural definition of two independent Wiener processes $W^{(1)}$ and $W^{(2)}$ in terms of a single Wiener process $W$.

From SDEs (E.5) one obtains, applying the Itô formula [5] to the function $r=\sqrt{x^{2}+y^{2}}$,

$$
\begin{aligned}
\mathrm{d} r_{t}= & {\left[\frac{\tau}{2}\left(1-\frac{\delta}{\tau}\right) \frac{v}{r_{t}}\left(x_{t} \frac{\partial v}{\partial x}+y_{t} \frac{\partial v}{y}\right)+\frac{\tau v^{2}}{r_{t}}\right] \mathrm{d} t } \\
& +\frac{\sqrt{\tau} v}{r_{t}}\left(x_{t} \mathrm{~d} W^{(1)}+y_{t} \mathrm{~d} W_{t}^{2)}\right) .
\end{aligned}
$$

Let us note that in the radially symmetric case, in which $v(x, y)=v(r)$ we may obtain from here after some calculations (with an aid of the Lévy theorem [5, theorem 8.6.1]) the limiting equation for the evolution of the radial process

$$
\mathrm{d} r_{t}=\left[\frac{\tau}{2}\left(1-\frac{\delta}{\tau}\right) v\left(r_{t}\right) \frac{\mathrm{d} v}{\mathrm{~d} r}\left(r_{t}\right)+\frac{\tau v\left(r_{t}\right)^{2}}{r_{t}}\right] \mathrm{d} B_{t},
$$

where the Wiener process $B$ is defined in terms of $W^{(1)}$ and $W^{(2)}$ by

$$
B_{t}=\int_{0}^{t}\left(\frac{x_{s}}{r_{s}} \mathrm{~d} W_{s}^{(1)}+\frac{y_{s}}{r_{s}} \mathrm{~d} W_{s}^{(2)}\right) .
$$

Going back to general case, we now use a standard method in diffusion theory to find the density of the stationary distribution of the stochastic evolution governed by the limiting SDEs (E.5). Namely, we consider the adjoint to the backward Kolmogorov equation (equation (E.3)), which is called forward Kolmogorov or Fokker-Planck equation:

$$
\begin{aligned}
\frac{\partial \rho_{0}}{\partial t}= & -\frac{\tau}{2}\left(1-\frac{\delta}{\tau}\right)\left[\frac{\partial}{\partial x}\left(v \frac{\partial v}{\partial x} \rho_{0}\right)+\frac{\partial}{\partial y}\left(v \frac{\partial v}{\partial y} \rho_{0}\right)\right] \\
& +\frac{\tau}{2} \Delta\left(v^{2} \rho_{0}\right) .
\end{aligned}
$$

This equation describes the time evolution of a probability density under the dynamics of the corresponding SDE system. The stationary density given by equation (33) was obtained by equating its right-hand side to zero.

\section{References}

[1] Strogatz S H 2014 Nonlinear Dynamics and Chaos: with Applications to Physics, Biology, Chemistry, and Engineering (Cambridge, MA: Westview Press)

[2] Nelson E 1967 Dynamical theories of Brownian motion (Princeton, NJ: Princeton University Press)

[3] Karatzas I and Shreve S 2012 Brownian Motion and Stochastic Calculus (New York: Springer)

[4] Kloeden P and Platen E 1992 Numerical Solutions of Stochastic Differential Equations (Berlin: Springer)

[5] Øksendal B 2013 Stochastic Differential Equations: an Introduction with Applications (Berlin: Springer)

[6] Pavliotis G 2014 Stochastic Processes and Applications (Berlin: Springer)

[7] Krumscheid S, Pradas M, Pavliotis G A and Kalliadasis S 2015 Data-driven coarse graining in action: modeling and prediction of complex systems Phys. Rev. E 92042139

[8] Klafter J and Sokolov I M 2011 First Steps in Random Walks: from Tools to Applications (Oxford: Oxford University Press)

[9] Brenner H 1961 The slow motion of a sphere through a viscous fluid towards a plane surface Chem. Eng. Sci. $16242-51$

[10] Cherstvy A G, Chechkin A V and Metzler R 2013 Anomalous diffusion and ergodicity breaking in heterogeneous diffusion processes New J. Phys. 15083039

[11] Cherstvy A G and Metzler R 2013 Population splitting, trapping, and non-ergodicity in heterogeneous diffusion processes Phys. Chem. Chem. Phys. 15 20220-35

[12] Lançon P, Batrouni G, Lobry L and Ostrowsky N 2001 Drift without flux: Brownian walker with a space-dependent diffusion coefficient Europhys. Lett. 54 28-34

[13] Hamao Y, Masulis R W and Ng V 1990 Correlations in price changes and volatility across international stock markets Rev. Financ. Stud. 3 281-307

[14] Kaern M, Elston T C, Blake W J and Collins J J 2005 Stochasticity in gene expression: From theories to phenotypes Nat. Rev. Genet. 6 451-64

[15] Sussmann H J 1978 On the gap between deterministic and stochastic ordinary differential equations Ann. Prob. $6019-41$

[16] van Kampen N G 1981 Itô versus Stratonovich J. Stat. Phys. 24 175-87

[17] Itô K 1944 Stochastic integral Proc. Imp. Acad. 20 519-24

[18] Stratonovich R L 1966 A new representation for stochastic integrals and equations SIAM J. Control 4 362-71 
[19] Hänggi P 1982 Nonlinear fluctuations: the problem of deterministic limit and reconstruction of stochastic dynamics Phys. Rev. A 25 1130-6

[20] Klimontovich Yu L 1990 Itô, Stratonovich and kinetic forms of stochastic equations Physica A 163 515-32

[21] Turelli M 1977 Random environments and stochastic calculus Theor. Pop. Biol. 12 140-78

[22] Smythe J, Moss F and McClintock P V E 1983 Observation of a noise-induced phase transition with an analog simulator Phys. Rev. Lett. 51 1062-5

[23] Wong E and Zakai M 1969 Riemann-Stieltjes approximations of stochastic integrals Z. Wahr. Verw. Geb. 12 87-97

[24] Ermak D L and McCammon J A 1978 Brownian dynamics with hydrodynamic interactions J. Chem. Phys. 69 1352-60

[25] Lau A W C and Lubensky T C 2007 State-dependent diffusion: thermodynamic consistency and its path integral formulation Phys. Rev. E 76011123

[26] Volpe G, Helden L, Brettschneider T, Wehr J and Bechinger C 2010 Influence of noise on force measurements Phys. Rev. Lett. 104170602

[27] Pesce G, McDaniel A, Hottovy S, Wehr J and Volpe G 2013 Stratonovich-to-Itô transition in noisy systems with multiplicative feedback Nat. Commun. 42733

[28] Mannella R and McClintock P V E 2011 Comment on influence of noise on force measurements Phys. Rev. Lett. 107078901

[29] Volpe G, Helden L, Brettschneider T, Wehr J and Bechinger C 2011 Reply to comment on 'Influence of noise on force measurements' Phys. Rev. Lett. 107078902

[30] Yang M and Ripoll M 2012 Drift velocity in non-isothermal inhomogeneous systems J. Chem. Phys. 136204508

[31] Farago O and Grønbech-Jensen N 2014 Langevin dynamics in inhomogeneous media: re-examining the Itô-Stratonovich dilemma Phys. Rev. E 89013301

[32] Massignan P, Manzo C, Torreno-Pina J A, García-Parajo M F, Lewenstein M and Lapeyre G J 2014 Nonergodic subdiffusion from Brownian motion in an inhomogeneous medium Phys. Rev. Lett. 112150603

[33] Srokowski T 2015 Anomalous diffusion in stochastic systems with nonhomogeneously distributed traps Phys. Rev. E 91052141

[34] Durang X, Kwon C and Park H 2015 Overdamped limit and inverse-friction expansion for Brownian motion in an inhomogeneous medium Phys. Rev. E 91062118

[35] Kazakevičius R and Ruseckas J 2015 Power law statistics in the velocity fluctuations of Brownian particle in inhomogeneous media and driven by colored noise J. Stat. Mech. P02021

[36] Manzo C, Torreno-Pina J A, Massignan P, Lapeyre G J, Lewenstein M and Garcia Parajo M F 2015 Weak ergodicity breaking of receptor motion in living cells stemming from random diffusivity Phys. Rev. X 5011021

[37] Massignan P, Lampo A, Wehr J and Lewenstein M 2015 Quantum Brownian motion with inhomogeneous damping and diffusion Phys. Rev. A 91033627

[38] Ryter D 1981 Brownian motion in inhomogeneous media and with interacting particles Z. Phys. B: Condens. Matter 41 39-42

[39] Sancho J M, San Miguel M and Dürr D 1982 Adiabatic elimination for systems of Brownian particles with nonconstant damping coefficients J. Stat. Phys. 28 291-305

[40] Lançon P, Batrouni G, Lobry L and Ostrowsky N 2002 Brownian walker in a confined geometry leading to a spacedependent diffusion coefficient Physica A 304 65-76

[41] Brettschneider T, Volpe G, Helden L, Wehr J and Bechinger C 2011 Force measurement in the presence of Brownian noise: equilibrium-distribution method versus drift method Phys. Rev. E 83041113
[42] Zwanzig R 2001 Nonequilibrium Statistical Mechanics (Oxford: Oxford University Press)

[43] Li T and Raizen M G 2013 Brownian motion at short time scales Ann. Phys. 525 281-95

[44] Volpe G, Brettschneider T, Helden L and Bechinger C 2009 Novel perspectives for the application of total internal reflection microscopy Opt. Express 17 23975-85

[45] Beltran-Villegas D J, Edwards T D and Bevan M A 2013 Selfconsistent colloidal energy and diffusivity landscapes in macromolecular solutions Langmuir 29 12337-41

[46] Ao P, Kwon C and Qian H 2007 On the existence of potential landscape in the evolution of complex systems Complexity 12 19-27

[47] Hottovy S, Volpe G and Wehr J 2012 Noise-induced drift in stochastic differential equations with arbitrary friction and diffusion in the Smoluchowski-Kramers limit J. Stat. Phys. $146762-73$

[48] Hottovy S, McDaniel A, Volpe G and Wehr J 2015 The Smoluchowski-Kramers limit of stochastic differential equations with arbitrary state-dependent friction Commun. Math. Phys. 336 1259-83

[49] Hottovy S, Volpe G and Wehr J 2012 Thermophoresis of Brownian particles driven by coloured noise Europhys. Lett. 9960002

[50] Smoluchowski M 1916 Drei vortrage über Diffusion, Brownsche Bewegung und Koagulation von Kolloidteilchen Phys. Z. 17 557-85

[51] Kramers H A 1940 Brownian motion in a field of force and the diffusion model of chemical reactions Physica 7 284-304

[52] Schuss Z 1980 Theory and Applications of Stochastic Differential Equations (New York: Wiley)

[53] Papanicolaou G C 1977 Introduction to the asymptotic analysis of stochastic equations Modern Modeling of Continuum Phenomena (9th Summer Seminar on Applied Mathematics (Rensselaer Polytechnic Institute, Troy NY, 1975)) (Lectures in Applied Mathematics vol 16) (Providence, RI: American Mathematical Society) pp 109-47

[54] Pavliotis G A and Stuart A 2008 Multiscale methods: Averaging and homogenization (Berlin: Springer)

[55] Pardoux E and Veretennikov A Yu 2003 On Poisson equation and diffusion approximation. I,II,III Ann. Probab. 31 1166-92

[56] Freidlin M 2004 Some remarks on the SmoluchowskiKramers approximation J. Stat. Phys. 117 617-34

[57] Wong E and Zakai M 1965 On the convergence of ordinary integrals to stochastic integrals Ann. Math. Stat. 36 1560-4

[58] Kurtz T G and Protter P 1991 Weak limit theorems for stochastic integrals and stochastic differential equations Ann. Probab. 19 1035-70

[59] Kupferman R, Pavliotis G A and Stuart A M 2004 Itô versus Stratonovich white-noise limits for systems with inertia and colored multiplicative noise Phys. Rev. E 70036120

[60] Freidlin M and Hu W 2011 Smoluchowski-Kramers approximation in the case of variable friction J. Math. Sci. 179 184-207

[61] McDaniel A, Duman O, Volpe G and Wehr J 2016 An SDE approximation for stochastic differential delay equations with colored state-dependent noise Markov Proc. Rel. Fields to appear (arXiv:1406.7287)

[62] Mijalkov M, McDaniel A, Wehr J and Volpe G 2016 Engineering sensorial delay to control phototaxis and emergent collective behaviors Phys. Rev. X 6011008

[63] Ebbens S J and Howse J R 2010 In pursuit of propulsion at the nanoscale Soft Matter 6 726-38

[64] Volpe G, Gigan S and Volpe G 2014 Simulation of the active Brownian motion of a microswimmer Am. J. Phys. 82 659-64 
[65] Yuan R, Wang X, Ma Y, Yuan B and Ao P 2013 Exploring a noisy van der Pol type oscillator with a stochastic approach Phys. Rev. E 87062109

[66] Tang Y, Yuan R and Ma Y 2013 Dynamical behaviors determined by the Lyapunov function in competitive Lotka-Volterra systems Phys. Rev. E 87012708

[67] Arnold L 2013 Random Dynamical Systems (Berlin: Springer)

[68] Arnold L 1990 Stabilization by noise revisited Z. Angew. Math. Mech. 70 235-46

[69] Arenas Z G and Barci D G 2012 Hidden symmetries and equilibrium properties of multiplicative white-noise stochastic processes J. Stat. Mech. P12005

[70] Farago O and Grønbech-Jensen N 2014 Fluctuationdissipation relation for systems with spatially varying friction J. Stat. Phys. 156 1093-110

[71] Tang Y, Yuan R and Ao P 2014 Nonequilibrium work relation beyond the Boltzmann-Gibbs distribution Phys. Rev. E 89062112

[72] Gammaitoni L, Hänggi P, Jung P and Marchesoni F 1998 Stochastic resonance Rev. Mod. Phys. 70 223-87

[73] Faucheux L P, Bourdieu L S, Kaplan P D and Libchaber A J 1995 Optical thermal ratchet Phys. Rev. Lett. 74 1504-7

[74] Chetrite R and Gawędzki K 2008 Fluctuation relations for diffusion processes Commun. Math. Phys. 282 469-518

[75] Celani A, Bo S, Eichhorn R and Aurell E 2012 Anomalous thermodynamics at the microscale Phys. Rev. Lett. 109260603

[76] Polettini M 2013 Diffusion in nonuniform temperature and its geometric analog Phys. Rev. E 87032126

[77] Polettini M 2013 Generally covariant state-dependent diffusion J. Stat. Mech. P07005
[78] Li X-M 2016 Random perturbation to the geodesic equation Ann. Prob. to appear (arXiv:1402.5861)

[79] Nelson E 1985 Quantum Fluctuations (Princeton, NJ: Princeton University Press)

[80] Hsu E P 2002 Stochastic Analysis on Manifolds (Providence, RI: American Mathematical Society)

[81] Chepizhko O and Peruani F 2013 Diffusion, subdiffusion, and trapping of active particles in heterogeneous media Phys. Rev. Lett. 111160604

[82] Herzog D P, Hottovy S and Volpe G 2015 The smallmass limit for Langevin dynamics with unbounded coefficients and positive friction J. Stat. Phys. to appear (arXiv:1510.04187)

[83] Marchesoni F 2013 Drift in diffusion gradients Materials $63598-609$

[84] Bellman R E 1970 Introduction to Matrix Analysis (New York: McGraw-Hill)

[85] Schimansky-Geier L and Zülicke Ch 1990 Harmonic noise: effect on bistable systems Z. Phys. B: Condens. Matter 79 451-60

[86] Billingsley P 1999 Convergence of Probability Measures 2nd edn (New York: Wiley)

[87] Volpe G and Volpe G 2013 Simulation of a Brownian particle in an optical trap Am. J. Phys. 81 224-30

[88] Franosch T, Grimm M, Belushkin M, Mor F M, Foffi G, Forró L and Jeney S 2011 Resonances arising from hydrodynamic memory in Brownian motion Nature 478 85-8

[89] Pesce G, Volpe G, Volpe G and Sasso A 2014 Long-term influence of fluid inertia on the diffusion of a Brownian particle Phys. Rev. E 90042309

[90] Hottovy S, McDaniel A and Wehr J 2015 A small delay and correlation time limit of stochastic differential delay equations with state-dependent colored noise arXiv: 1510.05065 